\title{
HANKEL OPERATORS ON FOCK SPACES AND RELATED BERGMAN KERNEL ESTIMATES
}

\author{
KRISTIAN SEIP AND EL HASSAN YOUSSFI
}

\begin{abstract}
Hankel operators with anti-holomorphic symbols are studied for a large class of weighted Fock spaces on $\mathbb{C}^{n}$. The weights defining these Hilbert spaces are radial and subject to a mild smoothness condition. In addition, it is assumed that the weights decay at least as fast as the classical Gaussian weight. The main result of the paper says that a Hankel operator on such a Fock space is bounded if and only if the symbol belongs to a certain BMOA space, defined via the Berezin transform. The latter space coincides with a corresponding Bloch space which is defined by means of the Bergman metric. This characterization of boundedness relies on certain precise estimates for the Bergman kernel and the Bergman metric. Characterizations of compact Hankel operators and Schatten class Hankel operators are also given. In the latter case, results on Carleson measures and Toeplitz operators along with Hörmander's $L^{2}$ estimates for the $\bar{\partial}$ operator are key ingredients in the proof.
\end{abstract}

\section{INTRODUCTION}

This paper presents the basics of Hankel operators with anti-holomorphic symbols for a large class of weighted Fock spaces. Thus certain natural analogues of BMOA, the Bloch space, the little Bloch space, and the Besov spaces are identified and shown to play similar roles as their classical counterparts do. We will see that these spaces contain all holomorphic polynomials and are infinite-dimensional whenever the weight decays so fast that there exist functions of infinite order belonging to the Fock space.

The setting is the following. Consider a $C^{3}$-function $\Psi:[0,+\infty[\rightarrow[0,+\infty[$ such that

$$
\Psi^{\prime}(x)>0, \quad \Psi^{\prime \prime}(x) \geq 0, \text { and } \Psi^{\prime \prime \prime}(x) \geq 0 .
$$

We will refer to such a function as a logarithmic growth function. Note that (1.1) effectively says that $\Psi$ should grow at least as a linear function. Set

$$
d \mu_{\Psi}(z):=e^{-\Psi\left(|z|^{2}\right)} d V(z),
$$

where $d V$ denotes Lebesgue measure on $\mathbb{C}^{n}$, and let $\mathcal{A}^{2}(\Psi)$ be the Fock space defined as the closure of the set of holomorphic polynomials in $L^{2}\left(\mu_{\Psi}\right)$. We observe that $\mathcal{A}^{2}(\Psi)$ coincides with the classical Fock space when $\Psi$ is a suitably normalized linear function.

2000 Mathematics Subject Classification. Primary 47B35, 32A36, 32A37.

Key words and phrases. Bergman kernel, Hankel operator, Fock space.

The first author is supported by the Research Council of Norway grant 185359/V30. The second author is supported by the French ANR DYNOP, Blanc07-198398. 
It is immediate that

$$
s_{d}:=\int_{0}^{+\infty} x^{d} e^{-\Psi(x)} d x<+\infty
$$

for all nonnegative integers $d$. Moreover, as shown in [9], the series

$$
F_{s}(\zeta):=\sum_{d=0}^{+\infty} \frac{\zeta^{d}}{s_{d}}, \zeta \in \mathbb{C},
$$

has an infinite radius of convergence and $\mathcal{A}^{2}(\Psi)$ is a reproducing kernel Hilbert space with reproducing kernel

$$
K_{\Psi}(z, w)=\frac{1}{(n-1) !} F_{s}^{(n-1)}(\langle z, w\rangle), \quad z, w \in \mathbb{C}^{n} .
$$

This implies that the orthogonal projection $P_{\Psi}$ from $L^{2}\left(\mu_{\Psi}\right)$ onto $\mathcal{A}^{2}(\Psi)$ can be expressed as

$$
\left(P_{\Psi} g\right)(z)=\int_{\mathbb{C}^{n}} K_{\Psi}(z, w) g(w) d \mu_{\Psi}(w), z \in \mathbb{C}^{n},
$$

for every function $g$ in $L^{2}\left(\mu_{\Psi}\right)$. The domain of this integral operator can be extended to include functions $g$ that satisfy $K_{\Psi}(z, \cdot) g \in L^{1}\left(\mu_{\Psi}\right)$ for every $z$ in $\mathbb{C}^{n}$. This extension allows us to define (big) Hankel operators. To do so, denote by $\mathcal{T}(\Psi)$ the class of all $f$ in $L^{2}\left(\mu_{\Psi}\right)$ such that $f \varphi K_{\Psi}(z, \cdot) \in L^{1}\left(\mu_{\Psi}\right)$ for all holomorphic polynomials $\varphi$ and $z$ in $\mathbb{C}^{n}$ and the function

$$
H_{f}(\varphi)(z):=\int_{\mathbb{C}^{n}} K_{\Psi}(z, w) \varphi(w)[f(z)-f(w)] d \mu_{\Psi}(w), \quad z \in \mathbb{C}^{n},
$$

is in $L^{2}\left(\mu_{\Psi}\right)$. This is a densely defined operator from $\mathcal{A}^{2}(\Psi)$ into $L^{2}\left(\mu_{\Psi}\right)$ which will be called the Hankel operator $H_{f}$ with symbol $f$. It can be written in the form

$$
H_{f}(\varphi)=\left(I-P_{\Psi}\right)(f \varphi)
$$

for all holomorphic polynomials $\varphi$. It is clear that the class $\mathcal{T}(\Psi)$ contains all holomorphic polynomials.

Our main theorem involves the analogues in our setting of the space BMOA and the Bloch space. The analogue of BMOA is most conveniently defined via the Berezin transform, which for a linear operator $T$ on $\mathcal{A}^{2}(\Psi)$ is the function $\widetilde{T}$ defined on $\mathbb{C}^{n}$ by

$$
\widetilde{T}(z):=\frac{\left\langle T K_{\Psi}(\cdot, z), K_{\Psi}(\cdot, z)\right\rangle}{K_{\Psi}(z, z)} .
$$

If $T=M_{f}$ is the operator of multiplication by the function $f$, then we just set $\widetilde{M}_{f}=\tilde{f}$. We set

$$
\|f\|_{\mathrm{BMO}}:=\sup _{z \in \mathbb{C}^{n}}(\mathrm{MO} f)(z)
$$

where

$$
(\operatorname{MOf})(z):=\sqrt{\widetilde{|f|^{2}}(z)-|\tilde{f}(z)|^{2}}
$$

and define $\operatorname{BMO}(\Psi)$ as the set of functions $f$ on $\mathbb{C}^{n}$ for which $\widetilde{|f|^{2}}(z)$ is finite for every $z$ and $\|f\|_{\mathrm{BMO}}<\infty$. It is plain that $\operatorname{BMO}(\Psi)$ is a subset of $\mathcal{T}(\Psi)$. The space $\operatorname{BMOA}(\Psi)$ is the 
subspace of $\operatorname{BMO}(\Psi)$ consisting of analytic elements; this space is in turn a subset of $\mathcal{T}(\Psi) \cap$ $\mathcal{A}^{2}(\Psi)$.

We next introduce the Bergman metric associated with $\Psi$. To this end, set $\Lambda_{\Psi}(z)=\log K_{\Psi}(z, z)$ and

$$
\beta^{2}(z, \xi):=\sum_{j, k=1}^{n} \frac{\partial^{2} \Lambda_{\Psi}(z)}{\partial z_{j} \partial \bar{z}_{k}} \xi_{j} \bar{\xi}_{k}
$$

for arbitrary vectors $z=\left(z_{1}, \ldots, z_{n}\right)$ and $\xi=\left(\xi_{1}, \ldots, \xi_{n}\right)$ in $\mathbb{C}^{n}$. The corresponding distance $\varrho$ is given by

$$
\varrho(z, w):=\inf _{\gamma} \int_{0}^{1} \beta\left(\gamma(t), \gamma^{\prime}(t)\right) d t,
$$

where the infimum is taken over all piecewise $C^{1}$-smooth curves $\gamma:[0,1] \rightarrow \mathbb{C}^{n}$ such that $\gamma(0)=z$ and $\gamma(1)=w$. We define the Bloch space $\mathcal{B}(\Psi)$ to be the space of all entire funtions $f$ such that

$$
\|f\|_{\mathcal{B}(\Psi)}:=\sup _{z \in \mathbb{C}^{n}}\left[\sup _{\xi \in \mathbb{C}^{n} \backslash\{0\}} \frac{|\langle(\nabla f)(z), \bar{\xi}\rangle|}{\beta(z, \xi)}\right]<+\infty .
$$

In what follows, the function

$$
\Phi(x):=x \Psi^{\prime}(x)
$$

will play a central role. By (1.1), we have that both $\Phi^{\prime}(x)>0$ and $\Phi^{\prime \prime}(x)>0$, and it may be checked that $\Phi^{\prime}\left(|z|^{2}\right)$ coincides with the Laplacian of $\Psi\left(|z|^{2}\right)$ when $n=1$ and in general is bounded below and above by positive constants times this Laplacian for arbitrary $n>1$.

We are now prepared to state our main result.

Theorem A. Let $\Psi$ be a logarithmic growth function, and suppose that there exists a real number $\eta<1 / 2$ such that

$$
\Phi^{\prime \prime}(t)=O\left(t^{-\frac{1}{2}}\left[\Phi^{\prime}(t)\right]^{1+\eta}\right) \text { when } t \rightarrow \infty .
$$

If $f$ is an entire function on $\mathbb{C}^{n}$, then the following statements are equivalent:

(i) The function $f$ belongs to $\mathcal{T}(\Psi)$ and the Hankel operator $H_{\bar{f}}$ on $\mathcal{A}^{2}(\Psi)$ is bounded;

(ii) The function $f$ belongs to $\operatorname{BMOA}(\Psi)$;

(iii) The function $f$ belongs to $\mathcal{B}(\Psi)$.

Note that the additional assumption (1.4) is just a mild smoothness condition, which holds whenever $\Psi$ is a nontrivial polynomial or a reasonably well-behaved function of super-polynomial growth.

As part of the proof of Theorem A, we will perform a precise computation of the asymptotic behavior of $\beta(z, \xi)$ when $|z| \rightarrow \infty$. We state this result as a separate theorem.

Theorem B. Let $\Psi$ be a logarithmic growth function, and suppose that there exists a real number $\eta<1 / 2$ such that (1.4) holds. Then we have, uniformly in $\xi$, that

$$
\beta^{2}(z, \xi)=(1+o(1))\left(|\xi|^{2} \Psi^{\prime}\left(|z|^{2}\right)+|\langle z, \xi\rangle|^{2} \Psi^{\prime \prime}\left(|z|^{2}\right)\right) \text { when }|z| \rightarrow \infty .
$$


We observe that for the classical Fock space ( $\Psi$ a linear function) we have $\Psi^{\prime \prime}(x) \equiv 0$, and so the "directional" term in $\beta(z, \xi)$ is not present. Note also that $\mathcal{B}(\Psi)$ contains all polynomials and is infinite-dimensional whenever the growth of $\Psi^{\prime}(x)$ is super-polynomial. In the language of entire functions, this means that $\mathcal{A}^{2}(\Psi)$ contains functions of infinite order. When $n=1$, $\beta^{2}(z, \xi)$ can be replaced by $\Phi^{\prime}\left(|z|^{2}\right)|\xi|^{2}$. The same is also true when $\Psi$ is a polynomial, because then $\Psi^{\prime}$ and $\Phi^{\prime}$ have the same asymptotic behavior. In the latter case, our two theorems give the following precise result: If $\Psi$ is a polynomial of degree $d$, then $\mathcal{B}(\Psi)$ consists of all holomorphic polynomials of degree at most $d$, cf. Theorem A in [9].

The implication (i) $\Rightarrow$ (ii) in Theorem A is standard; it follows from general arguments for reproducing kernels. Likewise, the implication (ii) $\Rightarrow$ (iii) can be established by a well-known argument concerning the Bergman metric. Our proof of Theorem A (presented in sections 2-5 below) deals therefore mainly with the implication (iii) $\Rightarrow$ (i). The crucial technical ingredient in the proof of this result are certain estimates for the Bergman kernel $K_{\Psi}(z, w)$. Such estimates have previously been obtained by F. Holland and R. Rochberg in [11]. The results of [11] are not directly applicable because we need more precise off-diagonal estimates for the kernel than those given in that paper. Our method of proof is similar to that of [11], but our approach highlights more explicitly the interplay between the smoothness of $\Psi$ and the off-diagonal decay of the Bergman kernel. This is where the additional smoothness condition (1.4) comes into play; many of our estimates can be performed with sufficient precision without the assumption that (1.4) holds, but some condition of this kind seems to be needed for our off-diagonal estimates.

The fact that the Bergman metric is the notion used to define the Bloch space $\mathcal{B}(\Psi)$ suggests that Theorem A should be extendable beyond the case of radial weights. To obtain such an extension, one would need a replacement of our Fourier-analytic approach, which relies crucially on the representation of the Bergman kernel as a power series.

The machinery developed to prove Theorem A leads with little extra effort to a characterization of compact Hankel operators in terms of the obvious counterparts to VMOA and the little Bloch space; see Section 6 for details. In our study of Schatten class Hankel operators, however, some additional techniques will be used. We will need more precise local information about the Bergman metric, namely that balls of fixed radius in the Bergman metric are effectively certain ellipsoids in the Euclidean metric of $\mathbb{C}^{n}$ (see Section 7). These results appear to be of independent interest; in particular, they lead to a characterization of Carleson measures and in turn to a characterization of the spectral properties of Toeplitz operators (see Section 8). Building on these results and using $L^{2}$ estimates for the $\bar{\partial}$ operator, we obtain in Section 9 a characterization of Schatten class Hankel operators.

To place the present investigation in context, we close this introduction with a few words on the literature. Boundedness and compactness of Hankel operators with arbitrary symbols have previously been considered only for the classical Fock space ( $\Psi$ a linear function); see for example [1], [2], [5], [6], [17], [18]. The methods of these papers, relying on the transitive selfaction of the group $\mathbb{C}^{n}$, can not be extended beyond this special case. Hankel operators with anti-holomorphic symbols defined on more general weighted Fock spaces were studied recently in [9] and [8], where it was shown that anti-holomorphic polynomials do not automatically induce bounded Hankel operators. For Bergman kernel estimates in similar settings, we refer to [15] 
and [16]. We finally mention [13] and [3]; the first of these papers focuses on small Hankel operators and the Heisenberg group action, while the second deals with Hankel operators for the Bergman projection on smoothly bounded pseudoconvex domains in $\mathbb{C}^{n}$.

A word on notation: Throughout this paper, the notation $U(z) \lesssim V(z)$ (or equivalently $V(z) \gtrsim$ $U(z)$ ) means that there is a constant $C$ such that $U(z) \leq C V(z)$ holds for all $z$ in the set in question, which may be a space of functions or a set of numbers. If both $U(z) \lesssim V(z)$ and $V(z) \lesssim U(z)$, then we write $U(z) \simeq V(z)$.

\section{General Arguments: (i) $\Rightarrow$ (ii) And (ii) $\Rightarrow$ (iii) In TheOrem A}

The following standard argument shows that (i) implies (ii) in Theorem A. To begin with, we note that if $f$ is in $\mathcal{A}^{2}(\psi)$, then $\tilde{f}=f$. Moreover, by the definition of the reproducing kernel, a computation shows that

$$
\widetilde{|f|^{2}}(z)-|f(z)|^{2}=\int_{\mathbb{C}^{n}}|f(\xi)-f(z)|^{2} \frac{\left|K_{\Psi}(\xi, z)\right|^{2}}{K_{\Psi}(z, z)} d \mu_{\Psi}(\xi)=\frac{\left\|H_{\bar{f}} K_{\Psi}(\cdot, z)\right\|^{2}}{K_{\Psi}(z, z)} .
$$

Hence, if $H_{\bar{f}}$ is bounded, then $\|f\|_{\mathrm{BMO}}<+\infty$.

The implication (ii) $\Rightarrow$ (iii) is a consequence of the following lemma, the proof of which is exactly as the proof of Corollary 1 in [4] (see pp. 319-321 in that paper).

Lemma 2.1. Suppose that $f$ is in $\operatorname{BMOA}(\Psi)$. Then for every piecewise $C^{1}$-smooth curve $\gamma$ : $[0,1] \rightarrow \mathbb{C}^{n}$ we have

$$
\left|\frac{d}{d t}(f \circ \gamma)(t)\right| \leq 2 \sqrt{2} \beta\left(\gamma(t), \gamma^{\prime}(t)\right)(\operatorname{MO} f)(\gamma(t))
$$

If we choose $\gamma(t)=z+t \xi$, then we obtain

$$
\frac{|\langle(\nabla f)(z), \bar{\xi}\rangle|}{\beta(z, \xi)} \leq 2 \sqrt{2}(\operatorname{MO} f)(z)
$$

for all $z$ in $\mathbb{C}^{n}$ and $\xi$ in $\mathbb{C}^{n} \backslash\{0\}$.

\section{ESTIMATES FOR THE BERGMAN KERNEL AND SOME RELATED FUNCTIONS}

This section is a somewhat elaborate preparation for the proof of Theorem B and also the proof of the implication (iii) $\Rightarrow$ (i) in Theorem A.

Set

$$
\theta_{0}(r):=\left[r \Phi^{\prime}(r)\right]^{-1 / 2}
$$

The key estimates for the Bergman kernel are the following.

Lemma 3.1. Suppose that (1.4) holds. Let $z$ and $w$ be arbitrary points in $\mathbb{C}^{n}$ such that $\langle z, w\rangle \neq 0$, and write $\langle z, w\rangle=r e^{i \theta}$, where $r>0$ and $-\pi<\theta \leq \pi$. Then we have

$$
\frac{1}{\left[\Psi^{\prime}(r)\right]^{n-1}} \frac{\left|K_{\Psi}(z, w)\right|}{e^{\Psi(r)}} \lesssim \begin{cases}\Phi^{\prime}(r), & |\theta| \leq \theta_{0}(r) \\ r^{-3 / 2}\left[\Phi^{\prime}(r)\right]^{-1 / 2}|\theta|^{-3}, & |\theta|>\theta_{0}(r) .\end{cases}
$$


Moreover, there exists a positive constant c such that if $\theta<c \theta_{0}(r)$, then

$$
\left|K_{\Psi}(z, w)\right| \gtrsim \Phi^{\prime}(r)\left[\Psi^{\prime}(r)\right]^{n-1} e^{\Psi(r)} .
$$

We collect a few preliminary results.

Lemma 3.2. Let $\eta$ be as in Theorem A. Then, for any fixed $\alpha>\eta$, we have

$$
\sup _{|\tau| \leq t^{1 / 2}\left[\Phi^{\prime}(t)\right]^{-\alpha}} \Phi^{\prime}(t+\tau)=(1+o(1)) \Phi^{\prime}(t)
$$

when $t \rightarrow \infty$.

Proof. The proof is similar to the proof of Lemma 6 in [11]. By (1.4), $\left[\Phi^{\prime}(x)\right]^{-1-\eta} \Phi^{\prime \prime}(x)=$ $O\left(x^{-1 / 2}\right)$ when $x \rightarrow \infty$, which implies that

$$
\left|\left[\Phi^{\prime}(t+\tau)\right]^{-\eta}-\left[\Phi^{\prime}(t)\right]^{-\eta}\right|=|\tau| O\left(t^{-1 / 2} \tau\right)
$$

when $t \rightarrow \infty$. The result follows from this relation.

In order to estimate $\left|K_{\Psi}(z, w)\right|$, we need precise information about the moments $s_{d}$. To this end, note that the integrand of

$$
\int_{0}^{\infty} x^{t} e^{-\Psi(x)} d x
$$

attains its maximum at $x=\Phi^{-1}(t)$. Set

$$
h_{t}(x)=-t \log x+\Psi(x)-\left(-t \log \Phi^{-1}(t)+\Psi\left(\Phi^{-1}(t)\right)\right)
$$

and

we may then write

$$
I(t)=\int_{0}^{\infty} e^{-h_{t}(x)} d x
$$

$$
s_{d}=e^{d \log \Phi^{-1}(d)-\Psi\left(\Phi^{-1}(d)\right)} I(d) .
$$

We have the following precise estimate for $I(t)$.

Lemma 3.3. For the function $I(t)$, we have

$$
I(t)=(\sqrt{2 \pi}+o(1))\left[\frac{\Phi^{-1}(t)}{\Phi^{\prime}\left(\Phi^{-1}(t)\right)}\right]^{1 / 2}
$$

when $t \rightarrow \infty$.

Proof. Set $\tau(x)=\sqrt{x}\left[\Phi^{\prime}(x)\right]^{-\alpha}$, where $\eta<\alpha<1 / 2$. Since

$$
h_{t}^{\prime \prime}(x)=\frac{\Phi^{\prime}(x)}{x}+\frac{t}{x^{2}}-\frac{\Phi(x)}{x^{2}}=\frac{\Phi^{\prime}(x)}{x}+\frac{1}{x^{2}}\left[\Phi\left(\Phi^{-1}(t)\right)-\Phi(x)\right],
$$

we have, by Lemma 3.2

$$
h_{t}^{\prime \prime}(x)=h_{t}^{\prime \prime}\left(\Phi^{-1}(t)\right)(1+o(1))
$$

when $\left|x-\Phi^{-1}(t)\right| \leq \tau\left(\Phi^{-1}(t)\right)$. On the other hand, by the convexity of $h_{t}$, we then have

$$
\left|h_{t}(x)\right| \geq \frac{1}{2}\left(h_{t}^{\prime \prime}\left(\Phi^{-1}(t)\right)+o(1)\right) \tau\left(\Phi^{-1}(t)\right)\left|x-\Phi^{-1}(t)\right|
$$


for $\left|x-\Phi^{-1}(t)\right| \geq \tau\left(\Phi^{-1}(t)\right)$. Setting for simplicity

$$
c=h_{t}^{\prime \prime}\left(\Phi^{-1}(t)\right)=\frac{\Phi^{\prime}\left(\Phi^{-1}(t)\right)}{\Phi^{-1}(t)}
$$

we then get

$$
I(t)=\int_{|x| \leq \tau\left(\Phi^{-1}(t)\right)} e^{-\frac{1}{2}(c+o(1)) x^{2}} d x+E(t)
$$

where

$$
|E(t)| \leq 2 \int_{x \geq \tau\left(\Phi^{-1}(t)\right)} e^{-\frac{1}{2}(c+o(1)) \tau\left(\Phi^{-1}(t)\right) x} d x .
$$

Thus the result follows, since the integral in (3.1) can be estimated by the corresponding Gaussian integral from $-\infty$ to $\infty$.

In what follows, we will estimate a number of integrals in a similar fashion, using Lemma 3.2 to split the domain of integration. The integrands will be of the type $e^{-g_{t}(x)} S_{t}(x)$ and satisfy the following:

(I) $g_{t}$ attains its minimum at a point $x_{0}=x_{0}(t) \rightarrow \infty$ with $g_{t}^{\prime \prime}(x)=(1+o(1)) c$ for $\left|x-x_{0}\right| \leq \tau$ and $1 / \tau=o(c)$ when $t \rightarrow \infty$.

(II) For $\left|x-x_{0}\right| \leq \tau, S_{t}(x)$ can be estimated by a constant $C$ times $\left|x-x_{0}\right|^{m}$ for some positive integer $m$.

(III) When $\left|x-x_{0}\right| \geq \tau$ and $\left|x-x_{0}\right|$ grows, the function $e^{-g_{t}(x)} S_{t}(x)$ decays so fast that

$$
\int_{0}^{\infty} e^{-g_{t}(x)}\left|S_{t}(x)\right| d x=(1+o(1)) \int_{\left|x-x_{0}\right| \leq \tau} e^{-g_{t}(x)}\left|S_{t}(x)\right| d x .
$$

Taking into account the formula

$$
\int_{0}^{\infty} x^{m} e^{-\frac{1}{2} c x^{2}} d x=(c / 2)^{-(m+1) / 2} \int_{0}^{\infty} x^{m} e^{-x^{2}} d x
$$

we then arrive at the estimate

$$
\int_{0}^{\infty} e^{-h_{t}(x)} S_{t}(x) d x=O\left(C c^{-(m+1) / 2}\right)
$$

when $t \rightarrow \infty$.

We will at one point encounter a slightly different variant of this scheme, obtained by replacing (II) by the following:

(II') For $\left|x-x_{0}\right| \leq \tau$, we have $S(x)=(1+o(1))\left(x-x_{0}\right)$ when $t \rightarrow \infty$.

In this case, because of the symmetry around the point $x_{0}$, we get the slightly better estimate

$$
\int_{0}^{\infty} e^{-h_{t}(x)} S(x) d x=o\left(c^{-1}\right)
$$

when $t \rightarrow \infty$.

To avoid tedious repetitions, we will in what follows omit most of the details of such calculus arguments. We will briefly state that conditions (I), (II), (III) (or respectively (I), (II'), (III)) are satisfied and conclude that this leads to the estimate (3.3) (or respectively (3.4)). 
In the proof of the next lemma, we will use this scheme three times.

Lemma 3.4. We have

$$
\begin{aligned}
I^{\prime}(t) & =O\left(\left[\Phi^{-1}(t) \Phi^{\prime}\left(\Phi^{-1}(t)\right)\right]^{-1 / 2} I(t)\right) \\
I^{\prime \prime}(t) & =O\left(\left[\Phi^{-1}(t) \Phi^{\prime}\left(\Phi^{-1}(t)\right)\right]^{-1} I(t)\right) \\
I^{\prime \prime \prime}(t) & =O\left(\left[\Phi^{-1}(t) \Phi^{\prime}\left(\Phi^{-1}(t)\right)\right]^{-3 / 2} I(t)\right)
\end{aligned}
$$

when $t \rightarrow \infty$.

Proof. We begin by noting that $I^{\prime}$ can be computed in the following painless way:

$$
I^{\prime}(t)=\int_{0}^{\infty} \log \frac{x}{\Phi^{-1}(t)} e^{-h_{t}(x)} d x
$$

this holds because $h_{t}^{\prime}\left(\Phi^{-1}(t)\right)=0$. For the same reason, we get

$$
I^{\prime \prime}(t)=\int_{0}^{\infty}\left[-\frac{\left(\Phi^{(-1)}\right)^{\prime}(t)}{\Phi^{-1}(t)}+\left(\log \frac{x}{\Phi^{-1}(t)}\right)^{2}\right] e^{-h_{t}(x)} d x
$$

and

$$
I^{\prime \prime \prime}(t)=\int_{0}^{\infty}\left[-\left[\frac{\left(\Phi^{(-1)}\right)^{\prime}(t)}{\Phi^{-1}(t)}\right]^{\prime}-3 \frac{\left(\Phi^{(-1)}\right)^{\prime}(t)}{\Phi^{-1}(t)} \log \frac{x}{\Phi^{-1}(t)}+\left(\log \frac{x}{\Phi^{-1}(t)}\right)^{3}\right] e^{-h_{t}(x)} d x
$$

We use that $\left[\Phi^{-1}\right]^{\prime}(t)=1 / \Phi^{\prime}\left(\Phi^{-1}(t)\right.$, and then in (3.7) we also use the fact that

$$
\left[\frac{1}{\Phi^{\prime}\left(\Phi^{(-1)}(t)\right) \Phi^{-1}(t)}\right]^{\prime}=-\frac{\Phi^{\prime \prime}\left(\Phi^{-1}(t)\right)}{\left[\Phi^{\prime}\left(\Phi^{-1}(t)\right)\right]^{3} \Phi^{-1}(t)}-\frac{1}{\left[\Phi^{\prime}\left(\Phi^{-1}(t)\right) \Phi^{-1}(t)\right]^{2}}
$$

we apply condition (1.4) to the first term on the right-hand side. When we estimate the integrals in (3.5), (3.6), and (3.7), we use that

$$
\left|\log \frac{x}{\Phi^{-1}(t)}\right| \leq e \frac{\left|x-\Phi^{-1}(t)\right|}{\Phi^{-1}(t)}
$$

for $x \geq e^{-1} \Phi^{-1}(t)$ and that, say,

$$
\left|\log \frac{x}{\Phi^{-1}(t)}\right| \leq \log \frac{1}{\Phi^{-1}(t)}
$$

when $1 \leq x<e^{-1} \Phi^{-1}(t)$. In each case, the integrand satisfies conditions (I), (II), (III) with $g_{t}=h_{t}$, so that we may use (3.3). The desired results for $I^{\prime}, I^{\prime \prime}, I^{\prime \prime \prime}$ now follow from (3.3).

We will need similar estimates for the function

$$
L_{r}(t)=\exp \left(t \log r-t \log \Phi^{-1}(t)+\Psi\left(\Phi^{-1}(t)\right)\right),
$$

where $r$ is a positive parameter. 
Lemma 3.5. We have

$$
\begin{aligned}
L_{r}^{\prime}(t) & =\left(-\log \frac{\Phi^{-1}(t)}{r}\right) L_{r}(t) \\
L_{r}^{\prime \prime}(t) & =\left[\left(\log \frac{\Phi^{-1}(t)}{r}\right)^{2}-\frac{1}{\Phi^{\prime}\left(\Phi^{-1}(t)\right) \Phi^{-1}(t)}\right] L_{r}(t) ; \\
L_{r}^{\prime \prime \prime}(t) & =\left[\left(-\log \frac{\Phi^{-1}(t)}{r}\right)^{3}+\frac{3 \log \frac{\Phi^{-1}(t)}{r}}{\Phi^{\prime}\left(\Phi^{-1}(t)\right) \Phi^{-1}(t)}+O\left(\left[\Phi^{\prime}\left(\Phi^{-1}(t)\right) \Phi^{-1}(t)\right]^{-3 / 2}\right)\right] L_{r}(t)
\end{aligned}
$$

when $t \rightarrow \infty$.

Proof. The first and the second of these formulas are obtained by direct computation. We arrive at the estimate for the third derivative by again using (3.8) and then applying condition (1.4).

Proof of Lemma 3.1] We begin by recalling that

$$
K_{\Psi}(z, w)=k(\langle z, w\rangle),
$$

where

$$
k(\zeta):=\frac{1}{(n-1) !} \sum_{d=n-1}^{\infty} \frac{d(d-1) \cdots(d-n+2)}{s_{d}} \zeta^{d-n+1} .
$$

We set $\langle z, w\rangle=r e^{i \theta}$ and assume that $r>0$ and $|\theta| \leq \pi$. We may then write

$$
\frac{\langle z, w\rangle^{d}}{s_{d}}=\frac{L_{r}(d)}{I(d)} \exp (i d \theta)
$$

and hence

$$
\begin{aligned}
\langle z, w\rangle^{n-1} K_{\Psi}(z, w) & =r^{n-1} \exp (i(n-1) \theta) k\left(r e^{i \theta}\right) \\
& =\frac{1}{(n-1) !} \sum_{d=n-1}^{\infty} d(d-1) \cdots(d-n+2) \frac{L_{r}(d)}{I(d)} \exp (i d \theta) .
\end{aligned}
$$

Let $\Omega(t)$ be a function in $C^{3}(\mathbb{R})$ so that

$$
\Omega(t)=\frac{1}{(n-1) !} \frac{t(t-1) \cdots(t-n+2) L_{r}(t)}{I(t)}
$$

for $t \geq n-1$ and $\Omega(t)=0$ for $t \leq n-2$. Then the Poisson summation formula gives

$$
r^{n-1} \exp (i(n-1) \theta) k\left(r e^{i \theta}\right)=\sum_{j=-\infty}^{\infty} \widetilde{\Omega}(j)
$$

where

$$
\widetilde{\Omega}(j)=\int_{-\infty}^{\infty} \Omega(t) e^{i(2 \pi j+\theta) t} d t
$$

Integrating by parts, we obtain

$$
r^{n-1}\left|k\left(r e^{i \theta}\right)\right| \leq|\widetilde{\Omega}(0)|+\left\|\Omega^{\prime \prime \prime}\right\|_{1} \sum_{j=1}^{\infty} \frac{2}{(2 \pi)^{3}(j-1 / 2)^{3}} .
$$


Since

$$
|\widetilde{\Omega}(0)| \leq \min \left(\|\Omega\|_{1},|\theta|^{-3}\left\|\Omega^{\prime \prime \prime}\right\|_{1}\right),
$$

the proof of the first part of the lemma is complete if we can prove that

$$
\|\Omega\|_{1} \lesssim(\Phi(r))^{n-1} \Phi^{\prime}(r) e^{\Psi(r)}
$$

and

$$
\left\|\Omega^{\prime \prime \prime}\right\|_{1} \lesssim(\Phi(r))^{n-1} \frac{e^{\Psi(r)}}{r^{3 / 2} \sqrt{\Phi^{\prime}(r)}} .
$$

We first estimate $\|\Omega\|_{1}$. We write $L_{r}(t)=\exp \left(-g_{r}(t)\right)$ and claim that conditions (I), (II), (III) above hold. To see this, we observe that, by the first formula of Lemma 3.5, $L_{r}$ attains its maximum at $t=\Phi(r)$. Moreover, $g_{r}$ is a convex function and

$$
g_{r}^{\prime \prime}(t)=\frac{1}{\Phi^{\prime}\left(\Phi^{-1}(t)\right) \Phi^{-1}(t)} .
$$

Lemma 3.2 implies that

$$
g_{r}^{\prime \prime}(t)=(1+o(1)) g_{r}^{\prime \prime}(\Phi(r))
$$

when $|t-\Phi(r)| \leq \sqrt{r}\left[\Phi^{\prime}(r)\right]^{1-2 \alpha}$. The remaining details are carried out as in the proof of Lemma 3.3. Using (3.3) with $m=0$ and Lemma 3.3, we therefore get

$$
\begin{aligned}
\|\Omega\|_{1} & =|\Phi(r)(\Phi(r)-1) \cdots(\Phi(r)-n+2)| \frac{L_{r}(\Phi(r))}{I(\Phi(r))}(\sqrt{2 \pi}+o(1))\left[\Phi^{\prime}(r) r\right]^{1 / 2} \\
& =(1+o(1))(\Phi(r))^{n-1} \Phi^{\prime}(r) e^{\Psi(r)},
\end{aligned}
$$

which shows that 3.9) holds.

To arrive at (3.10), we need a pointwise estimate for $\Omega^{\prime \prime \prime}$. To simplify the writing, we set

$$
a=\left|\log \frac{\Phi^{-1}(t)}{r}\right| \text { and } b=\left[\Phi^{\prime}\left(\Phi^{-1}(t)\right) \Phi^{-1}(t)\right]^{-1 / 2} .
$$

Then using the Leibniz rule along with Lemma 3.4 and Lemma 3.5, we get

$$
\left|\Omega^{\prime \prime \prime}(t)\right| \lesssim\left(a^{3}+a^{2} b+a b^{2}+b^{3}\right) \Omega(t) .
$$

By a straightforward calculus argument, we verify that each of the terms in this expression satisfies (I), (II), and (III) above, again with $x_{0}=\Phi(r) \tau=\sqrt{r}\left[\Phi^{\prime}(r)\right]^{1-2 \alpha}$. We now use (3.3) to achieve the desired estimate for each of the terms $a^{m} b^{3-m} \Omega(t)$.

The previous proof also gives the second estimate when $\theta=0$, because then $\widetilde{\Omega}(0)=\|\Omega\|_{1}$. To prove it in general, we need to check that $k(r) \simeq\left|k\left(r e^{i \theta}\right)\right|$ when $|\theta| \leq c\left[r \Phi^{\prime}(r)\right]^{-1 / 2}$. To this end, note that

$$
\widetilde{\Omega}(0)=e^{i \theta \Phi(r)} \int_{-\infty}^{\infty} \Omega(t) e^{i \theta(t-\Phi(r))} d t
$$

which implies that

$$
|\widetilde{\Omega}(0)| \geq\|\Omega\|_{1}-\int_{-\infty}^{\infty} \Omega(t)|\theta||t-\Phi(r)| d t .
$$


The integral on the right is computed using (3.3) with $m=1$, and so we get

$$
|\widetilde{\Omega}(0)| \geq\|\Omega\|_{1}\left(1-C|\theta|\left[r \Phi^{\prime}(r)\right]^{1 / 2}\right) .
$$

Thus the second estimate in Lemma 3.1 holds for $c$ sufficiently small.

We close this section by proving some estimates for another function that will be important later. Set

$$
Q_{x}(r)=\frac{1}{2}\left(\Psi\left(r^{2}\right)+\Psi\left(x^{2}\right)\right)-\Psi(x r) .
$$

Lemma 3.6. Let $\alpha$ be a positive number such that $\eta<\alpha<1 / 2$, let $x_{1}$ and $x_{2}$ be the two points such that $x_{1}<x<x_{2}$ and

$$
\left|x-x_{1}\right|=\left|x-x_{2}\right|=\left[\Phi^{\prime}(x)\right]^{-\alpha},
$$

and set $c=\Psi^{\prime}(0)$. When $r \rightarrow \infty$, we have

$$
\begin{aligned}
& Q_{x}^{\prime \prime}(r)=(1+o(1)) \Phi^{\prime}\left(x^{2}\right), \quad x_{1} \leq r \leq x_{2} ; \\
& Q_{x}(r) \geq \frac{c}{4}(x-r)^{2}+\left(\frac{1}{4}+o(1)\right)\left[\Phi^{\prime}\left(x^{2}\right)\right]^{1-2 \alpha}, \quad r<x_{1} ; \\
& Q_{x}(r) \geq \frac{c}{4}(x-r)^{2}+\left(\frac{1}{4}+o(1)\right)\left[\Phi^{\prime}\left(r^{2}\right)\right]^{1-2 \alpha}, \quad r>x_{2} .
\end{aligned}
$$

Proof. We begin by noting that

$$
Q_{x}^{\prime}(r)=r \Psi^{\prime}\left(r^{2}\right)-x \Psi^{\prime}(x r)
$$

and

$$
Q_{x}^{\prime \prime}(r)=\Psi^{\prime}\left(r^{2}\right)+2 r^{2} \Psi^{\prime \prime}\left(r^{2}\right)-x^{2} \Psi^{\prime \prime}(x r) .
$$

We observe that for $x_{1} \leq r \leq x_{2}$ Lemma 3.2 applies:

$$
Q_{x}^{\prime \prime}(r)=\Phi^{\prime}\left(r^{2}\right)+r^{2} \Psi^{\prime \prime}\left(r^{2}\right)-x^{2} \Psi^{\prime \prime}(x r)=(1+o(1)) \Phi^{\prime}\left(x^{2}\right),
$$

and so we have established (3.12). For $r<x_{1}$, we use the following estimate:

$$
\begin{aligned}
Q_{x}(r) & \geq \frac{1}{2} \int_{r}^{x} \Psi^{\prime}\left(s^{2}\right)(s-x) d s+\frac{1}{2} \int_{x-\left[\Phi^{\prime}\left(x^{2}\right)\right]^{-\alpha}}^{x} \int_{x-\left[\Phi^{\prime}\left(x^{2}\right)\right]^{-\alpha}}^{t} Q_{x}^{\prime \prime}(u) d u d t \\
& \geq \frac{c}{4}(x-r)^{2}+\left(\frac{1}{4}+o(1)\right)\left[\Phi^{\prime}\left(x^{2}\right)\right]^{1-2 \alpha},
\end{aligned}
$$

where we used again Lemma 3.2 in the last step. Now observe that since $\Psi^{\prime \prime}(y)$ is a nondecreasing function, we have

$$
Q_{x}^{\prime \prime}(r) \geq \Phi^{\prime}\left(r^{2}\right)
$$

for $r \geq x$. We therefore obtain for $x>x_{2}$ :

$$
\begin{aligned}
Q_{x}(r) & \geq \frac{1}{2} \int_{x}^{r} \Psi^{\prime}\left(s^{2}\right)(s-x) d s+\frac{1}{2} \int_{r-\left[\Phi^{\prime}\left(r^{2}\right)\right]^{-\alpha}}^{r} \int_{r-\left[\Phi^{\prime}\left(r^{2}\right)\right]^{-\alpha}}^{t} Q_{x}^{\prime \prime}(u) d u d t \\
& \geq \frac{c}{4}(x-r)^{2}+\left(\frac{1}{4}+o(1)\right)\left[\Phi^{\prime}\left(r^{2}\right)\right]^{1-2 \alpha}
\end{aligned}
$$

where Lemma 3.2 is applied once more. Hence (3.14) also holds. 


\section{Proof of Theorem B: Computation of the Bergman metric}

We begin by recalling that

$$
K_{\Psi}(z, z)=k\left(r^{2}\right)
$$

where

$$
k(r)=\sum_{n=0}^{\infty} c_{d} r^{d}
$$

and

$$
c_{d}:=\frac{(d+1) \cdots(d+n-1)}{(n-1) ! s_{d+n-1}} .
$$

A computation shows that

$$
\beta^{2}(z, \xi):=|\xi|^{2} \frac{k^{\prime}\left(|z|^{2}\right)}{k\left(|z|^{2}\right)}+|\langle z, \xi\rangle|^{2}\left[\frac{k^{\prime \prime}\left(\left(|z|^{2}\right)\right)}{k\left(\left(|z|^{2}\right)\right)}-\left(\frac{k^{\prime}\left(|z|^{2}\right)}{k\left(|z|^{2}\right)}\right)^{2}\right] .
$$

Thus Theorem B is a consequence of the following lemma.

Lemma 4.1. Suppose that (1.4) holds. Then we have

$$
\begin{aligned}
\frac{k^{\prime}(r)}{k(r)} & =(1+o(1)) \Psi^{\prime}(r), \\
\left(\frac{k^{\prime}(r)}{k(r)}\right)^{\prime} & =(1+o(1)) \Psi^{\prime \prime}(r)+o(1) \frac{\Psi^{\prime}(r)}{r}
\end{aligned}
$$

when $r \rightarrow \infty$.

The proof of this lemma relies on the following estimates.

Lemma 4.2. Suppose that (1.4) holds and let the coefficients $c_{d}$ be as defined above. Then we have

$$
\begin{aligned}
\sum_{d=1}^{\infty} c_{d}(d-\Phi(r)) r^{d} & =o\left(\left[r \Phi^{\prime}(r)\right]^{1 / 2} k(r)\right), \\
\sum_{d=1}^{\infty} c_{d}(d-\Phi(r))^{2} r^{d} & =(1+o(1)) r \Phi^{\prime}(r) k(r) .
\end{aligned}
$$

when $r \rightarrow \infty$.

Proof. The proof is essentially the same as the proof for the diagonal estimates in Lemma 3.1 . The only difference is that we replace the function $\Omega(t)$ by respectively $(t-\Phi(r)) \Omega(t)$ and $(t-\Phi(r))^{2} \Omega(t)$. In the first case, we have a function that satisfies condition (II') in Section 3. This means that we may use (3.4) to arrive at (4.1). To establish (4.2), may we apply (3.2) with $m=2$ and take into account that we have the explicit factor $(t-\Phi(r))^{2}$ in front of $\Omega(t)$. 
Proof of Lemma 4.1 We write

$$
k^{\prime}(r)=\frac{\Phi(r)}{r}(k(r)+O(1))+\frac{1}{r} \sum_{d=1}^{\infty} c_{d}(d-\Phi(r)) r^{d} ;
$$

using Lemma4.2, we obtain

$$
\frac{k^{\prime}(r)}{k(r)}=(1+o(1)) \Psi^{\prime}(r)+o\left(\left[\frac{\Phi^{\prime}(r)}{r}\right]^{1 / 2}\right) .
$$

The desired estimate for $k^{\prime} / k$ follows because, in view of Lemma 3.2, we have

$$
\Phi(r) \geq \int_{r-r^{1 / 2}\left[\Phi^{\prime}(r)\right]^{-\alpha}}^{r} \Phi^{\prime}(t) d t=(1+o(1)) r^{1 / 2}\left[\Phi^{\prime}(r)\right]^{1-\alpha}
$$

for some $\alpha<1 / 2$.

To arrive at the second estimate, we first observe that

$$
\begin{aligned}
k^{\prime \prime}(r) & =\frac{\Phi(r)-1}{r}\left(k^{\prime}(r)+O(1)\right)+\frac{1}{r} \sum_{d=2}^{\infty} c_{d} d(d-\Phi(r)) r^{d-1} \\
& =\frac{\Phi(r)-1}{r}\left(k^{\prime}(r)+O(1)\right)+\frac{\Phi(r)}{r^{2}} \sum_{d=2}^{\infty} c_{d}(d-\Phi(r)) r^{d}+\frac{1}{r^{2}} \sum_{d=2}^{\infty} c_{d}(d-\Phi(r))^{2} r^{d} .
\end{aligned}
$$

Combining our expressions for $k^{\prime}$ and $k^{\prime \prime}$, we find that

$$
\begin{aligned}
k^{\prime \prime}(r) k(r)-\left(k^{\prime}(r)\right)^{2}= & \frac{k(r)}{r^{2}} \sum_{d=2}^{\infty} c_{d}(d-\Phi(r))^{2} r^{d}-\frac{1}{r^{2}}\left[\sum_{d=2}^{\infty} c_{d}(d-\Phi(r)) r^{d}\right]^{2} \\
& -\frac{k(r) k^{\prime}(r)}{r}+\Psi^{\prime}(r) O\left(k(r)+k^{\prime}(r)\right) .
\end{aligned}
$$

Using again Lemma 4.2 and the estimate already obtained for $k^{\prime} / k$, we get

$$
\left(\frac{k^{\prime}(r)}{k(r)}\right)^{\prime}=(1+o(1)) \frac{\Phi^{\prime}(r)}{r}-(1+o(1)) \frac{\Phi(r)}{r^{2}}
$$

from which the second estimate in Lemma 4.1 follows.

\section{HANKEL OPERATORS FROM BLOCH FUNCTIONS}

We finally turn to the proof that (iii) implies (i) in Theorem A. A different proof, using $L^{2}$ estimates for the $\bar{\partial}$ operator will be given in Section 9 below, subject to an additional mild smoothness condition on $\Psi$. The proof in Section 9 gives a more informative norm estimate, which will be crucial in our study of Schatten class Hankel operators. The proof to be given below has the advantage that it does not require $f$ to be holomorphic.

Using the reproducing formula, we find that

$$
H_{\bar{f}} g(z)=\int_{\mathbb{C}^{n}}(\overline{f(z)}-\overline{f(w)}) K_{\Psi}(z, w) g(w) d \mu_{\Psi}(w) .
$$


Therefore, by the definition of $\mathcal{B}(\Psi)$, we have

$$
\left|H_{\bar{f}} g(z)\right| \leq\|f\|_{\mathcal{B}(\Psi)} \int_{\mathbb{C}^{n}} \varrho(z, w) K_{\Psi}(z, w) g(w) d \mu_{\Psi}(w) .
$$

Thus it suffices to prove that the operator $A$ defined as

$$
A g(z)=\int_{\mathbb{C}^{n}} \varrho(z, w) K_{\Psi}(z, w) g(w) d \mu_{\Psi}(w)
$$

is bounded on $L^{2}\left(\mu_{\Psi}\right)$.

We shall use a standard technique known as Schur's test [20, p. 42]. Set

$$
H(z, w)=\varrho(z, w)\left|K_{\Psi}(z, w)\right| e^{-\frac{1}{2}\left(\Psi\left(|z|^{2}\right)+\Psi\left(|w|^{2}\right)\right)} .
$$

By the Cauchy-Scwharz inequality, we obtain

$$
|(A g)(z)|^{2} e^{-\Psi\left(|z|^{2}\right)} \lesssim \int_{\mathbb{C}^{n}} H(z, \zeta) d V(\zeta) \int_{\mathbb{C}^{n}} H(z, w)|g(w)|^{2} e^{-\Psi\left(|w|^{2}\right)} d V(w) .
$$

This means that the operator $A$ is bounded on $L^{2}\left(\mu_{\Psi}\right)$ if

$$
\sup _{z} \int_{\mathbb{C}^{n}} H(z, \zeta) d V(\zeta)<\infty
$$

We therefore set as our task to establish (5.1).

Without loss of generality, we may assume that $z=(x, 0, \ldots, 0)$ with $x>0$. We begin by estimating $\varrho(z, w)$. To this end, write $w=\left(w_{1}, \xi\right)$ with $\xi$ a vector in $\mathbb{C}^{n-1}$ and $w_{1}=r e^{i \theta}$ when $n>1$. Set $e_{1}=(1,0, \ldots, 0)$ and consider the three curves

$$
\begin{aligned}
& \gamma_{1}(t)=x e^{i t} e_{1}, \quad 0 \leq t \leq \theta, \\
& \gamma_{2}(t)=(x+t(r-x)) e^{i \theta} e_{1}, \quad 0 \leq t \leq 1, \\
& \gamma_{3}(t)=\left(r e^{i \theta}, t \xi\right), \quad 0 \leq t \leq 1,
\end{aligned}
$$

which together constitute a piecewise smooth curve from $z$ to $w$. (When $n=1, \gamma_{3}$ does not appear and can be neglected.) Note that

$$
\begin{aligned}
& \left|\left\langle\gamma_{1}(t), \gamma_{1}^{\prime}(t)\right\rangle\right|=\left|\gamma_{1}(t)\right|\left|\gamma_{1}^{\prime}(t)\right|=x^{2}, \\
& \left|\left\langle\gamma_{2}(t), \gamma_{2}^{\prime}(t)\right\rangle\right|=\left|\gamma_{2}(t)\right|\left|\gamma_{2}^{\prime}(t)\right|=(x+t(r-x))|x-r|, \\
& \left|\left\langle\gamma_{3}(t), \gamma_{3}^{\prime}(t)\right\rangle\right|=t|\xi|^{2} .
\end{aligned}
$$

By these observations and Theorem B, we get the following estimate:

$$
\begin{aligned}
\varrho(z, w) \lesssim & x|\theta|\left[\Phi^{\prime}\left(x^{2}\right)\right]^{1 / 2}+\left[\Phi^{\prime}\left(\max \left(x^{2}, r^{2}\right)\right)\right]^{1 / 2}|x-r| \\
& +|\xi|\left[\Psi^{\prime}\left(r^{2}+|\xi|^{2}\right)\right]^{1 / 2}+|\xi|^{2}\left[\Psi^{\prime \prime}\left(r^{2}+|\xi|^{2}\right)\right]^{1 / 2} .
\end{aligned}
$$

When estimating the last term on the right-hand side of this inequality, we will use that

$$
\left[\Psi^{\prime}(y)\right]^{2} \gtrsim \Psi^{\prime \prime}(y)
$$


which is a consequence of our assumptions (1.1) and (1.4). Indeed, assuming $\Psi^{\prime \prime}>0$, we have $y \Psi^{\prime \prime}(y) \simeq \Phi^{\prime}(y)$ since $\Psi^{\prime \prime}$ is a nondecreasing function. Thus (5.2) is equivalent to the following:

$$
\Phi(t) \gtrsim t^{1 / 2}\left[\Phi^{\prime}(t)\right]^{1 / 2} \text {. }
$$

We arrive at this estimate because

$$
\Phi(t)=\Phi(0)+\int_{0}^{t} \Phi^{\prime}(\tau) d \tau \geq \Phi(0)+(1+o(1)) t^{1 / 2}\left[\Phi^{\prime}(t)\right]^{1 / 2}
$$

where in the second step we used Lemma 3.2 with $\alpha=1 / 2$.

For $\zeta=|\zeta| e^{i \theta}$, we set

$$
h(\zeta)= \begin{cases}\Phi^{\prime}(|\zeta|) & |\theta| \leq \theta_{0}(|\zeta|) \\ |\zeta|^{-3 / 2}\left[\Phi^{\prime}(|\zeta|)\right]^{-1 / 2}|\theta|^{-3}, & |\theta|>\theta_{0}(|\zeta|)\end{cases}
$$

Using this notation and Lemma 3.1, we then obtain

$$
H(z, w) \lesssim \varrho(x, w) h\left(x r e^{i \theta}\right)\left[\Psi^{\prime}(x r)\right]^{n-1} e^{-\frac{1}{2}\left(\Psi\left(x^{2}\right)+\Psi\left(r^{2}+|\xi|^{2}\right)\right)-\Psi(x r)} .
$$

By Fubini's theorem, we may compute the integral in (5.1) by first integrating with respect to the vector $\xi$ over $\mathbb{C}^{n-1}$ and then taking an area integral with respect to the complex variable $w_{1}$ over $\mathbb{C}$. Since $y \mapsto \Psi\left(r^{2}+y^{2}\right)$ attains its maximum at $y=0$ and has a second derivative larger than $2 \Psi^{\prime}\left(r^{2}\right)$, we have that $\Psi\left(r^{2}+y^{2}\right)-\Psi\left(r^{2}\right) \geq \Psi^{\prime}\left(r^{2}\right) y^{2}$. Using spherical coordinates along with this fact, we find that

$$
\int_{\mathbb{C}^{n-1}} e^{-\Psi\left(r^{2}+|\xi|^{2}\right)} d V_{n-1}(\xi) \lesssim e^{-\Psi\left(r^{2}\right)}\left[\Psi^{\prime}\left(r^{2}\right)\right]^{-n+1}
$$

Similarly, using again spherical coordinates, we get

$$
\int_{\mathbb{C}^{n-1}} \Theta(r,|\xi|) e^{-\Psi\left(r^{2}+|\xi|^{2}\right)} d V_{n-1}(\xi)=C \int_{0}^{\infty} \Theta(r, y) y^{2 n-2} e^{-\Psi\left(r^{2}+y^{2}\right)} d y,
$$

where $C$ is the surface area of the unit sphere in $\mathbb{C}^{n-1}$ and $\Theta$ is any suitable function of two variables. From the estimate for $\varrho(z, w)$ and (5.2) we see that we are interested in the following two choices: (1) $\Theta(r, y)=y\left[\Psi^{\prime}\left(r^{2}+y^{2}\right)\right]^{1 / 2}$ and (2) $\Theta(r, y)=y^{2} \Psi\left(r^{2}+y^{2}\right)$. In case (1), we use the Cauchy-Schwarz inequality, so that we get

$$
\int_{\mathbb{C}^{n-1}}|\xi|\left[\Psi^{\prime}\left(r^{2}+|\xi|^{2}\right)\right]^{1 / 2} e^{-\Psi\left(r^{2}+|\xi|^{2}\right)} d V_{n-1}(\xi) \lesssim e^{-\Psi\left(r^{2}\right)}\left[\int_{0}^{\infty} y^{4 n-3} e^{-\left(\Psi\left(r^{2}+y^{2}\right)-\Psi\left(r^{2}\right)\right)} d y\right]^{1 / 2} .
$$

Estimating $\Psi\left(r^{2}+y^{2}\right)-\Psi\left(r^{2}\right)$ as above, we therefore get

$$
\int_{\mathbb{C}^{n-1}}|\xi|\left[\Psi^{\prime}\left(r^{2}+|\xi|^{2}\right)\right]^{1 / 2} e^{-\Psi\left(r^{2}+|\xi|^{2}\right)} d V_{n-1}(\xi) \lesssim e^{-\Psi\left(r^{2}\right)}\left[\Psi^{\prime}\left(r^{2}\right)\right]^{-n+1} .
$$

In case (2), we integrate by parts and get

$$
\int_{\mathbb{C}^{n-1}}|\xi|^{2} \Psi^{\prime}\left(r^{2}+|\xi|^{2}\right) e^{-\Psi\left(r^{2}+|\xi|^{2}\right)} d V_{n-1}(\xi) \lesssim \int_{0}^{\infty} y^{2 n-1} e^{-\Psi\left(r^{2}+y^{2}\right)} d y .
$$


We proceed as above and obtain

$$
\int_{\mathbb{C}^{n-1}}|\xi|^{2} \Psi^{\prime}\left(r^{2}+|\xi|^{2}\right) e^{-\Psi\left(r^{2}+|\xi|^{2}\right)} d V_{n-1}(\xi) \lesssim e^{-\Psi\left(r^{2}\right)}\left[\Psi^{\prime}\left(r^{2}\right)\right]^{-n+1}
$$

With $\sigma$ denoting Lebesgue measure on $\mathbb{C}$, we therefore get

$$
\int_{\mathbb{C}^{n}} H(z, w) d V(w) \lesssim \int_{\mathbb{C}} G(x, r, \theta)\left[\frac{\Psi^{\prime}(r x)}{\Psi^{\prime}\left(r^{2}\right)}\right]^{n-1} h\left(x r e^{i \theta}\right) e^{-Q_{x}(r)} d \sigma\left(r e^{i \theta}\right),
$$

where

$$
G(x, r, \theta)=x|\theta|\left[\Phi^{\prime}\left(x^{2}\right)\right]^{1 / 2}+\left[\Phi^{\prime}\left(\max \left(x^{2}, r^{2}\right)\right)\right]^{1 / 2}|x-r|+1
$$

and $Q_{x}$ is as defined by (3.11).

We now resort to polar coordinates; simple calculations show that

$$
\int_{-\pi}^{\pi} h\left(x r e^{i \theta}\right) d \theta \lesssim\left[\frac{\Phi^{\prime}(x r)}{x r}\right]^{1 / 2} \text { and } \int_{-\pi}^{\pi}|\theta| h\left(x r e^{i \theta}\right) d \theta \lesssim \frac{1}{x r}
$$

so that

$$
\int_{\mathbb{C}^{n}} H(z, w) d V(w) \lesssim \int_{0}^{\infty}\left(S_{x}(r)+T_{x}(r)\right) e^{-Q_{x}(r)} r d r
$$

where

$$
S_{x}(r)=\left(\frac{\left[\Phi^{\prime}\left(x^{2}\right)\right]^{1 / 2}}{r}+\left[\frac{\Phi^{\prime}(x r)}{x r}\right]^{1 / 2}\right)\left[\frac{\Psi^{\prime}(r x)}{\Psi^{\prime}\left(r^{2}\right)}\right]^{n-1}
$$

and

$$
T_{x}(r)=\varphi\left(\max \left(x^{2}, r^{2}\right)\right)|x-r|\left[\frac{\Phi^{\prime}(x r)}{x r}\right]^{1 / 2}\left[\frac{\Psi^{\prime}(r x)}{\Psi^{\prime}\left(r^{2}\right)}\right]^{n-1} .
$$

By Lemma 3.6 and a straightforward argument, we find that both $S_{x} e^{-Q_{x}}$ and $T_{x} e^{-Q_{x}}$ satisfy conditions (I), (II), (III) of Section 3 (with $x=t, Q_{x}=g_{t}, x_{0}=x$, and $\tau=\left[\Phi^{\prime}(x)\right]^{-\alpha}$ ). Hence (3.3) applies with $m=0$ and $m=1$ for the respective integrands, so that we get

$$
\sup _{x>0} \int_{0}^{\infty} S_{x}(r) e^{-Q_{x}(r)} r d r<\infty \text { and } \sup _{x>0} \int_{0}^{\infty} T_{x}(r) e^{-Q_{x}(r)} r d r<\infty .
$$

We may therefore conclude that (5.1) holds.

\section{COMPACTNESS OF HANKEl OPERATORS}

We now turn to a study of the relation between the spectral properties of Hankel operators and the asymptotic behavior of their symbols. We begin with the case of compact Hankel operators.

An entire function is said to be of vanishing mean oscillation with respect to $\Psi$ if $(\mathrm{MO} f)(z)=$ $o(1)$ as $|z| \rightarrow+\infty$. Entire functions of vanishing mean oscillation form a closed subspace of $\operatorname{BMOA}(\Psi)$ which we will denote by $\operatorname{VMOA}(\Psi)$. In accordance with our preceding discussion, we define the little Bloch space $\mathcal{B}_{0}(\Psi)$ as the collection of functions $f$ in $\mathcal{B}(\Psi)$ for which

$$
\sup _{\xi \in \mathbb{C}^{n} \backslash\{0\}} \frac{|\langle\nabla f(z), \bar{\xi}\rangle|}{\beta(z, \xi)}=o(1) \text { when }|z| \rightarrow+\infty .
$$

The main result of this section reads as follows. 
Theorem C. Let $\Psi$ be a logarithmic growth function, and suppose that there exists a real number $\eta<1 / 2$ such that (1.4) holds. If $f$ is an entire function on $\mathbb{C}^{n}$, then the following statements are equivalent:

(i) The function $f$ belongs to $\mathcal{T}(\Psi)$ and the Hankel operator $H_{\bar{f}}$ on $\mathcal{A}^{2}(\Psi)$ is compact;

(ii) The function $f$ belongs to $\operatorname{VMOA}(\Psi)$;

(iii) The function $f$ belongs to $\mathcal{B}_{0}(\Psi)$.

Our proof of Theorem $\mathrm{C}$ requires the following two lemmas.

Lemma 6.1. The normalized Bergman kernels $K_{\Psi}(\cdot, z) / \sqrt{K_{\Psi}(z, z)}$ converge weakly to 0 in $\mathcal{A}^{2}(\Psi)$ when $|z| \rightarrow+\infty$.

Proof. Since the holomorphic polynomials are dense in $\mathcal{A}^{2}(\Psi)$, it suffices to show that for any non-negative integer $m$, we have

$$
\frac{|z|^{m}}{\sqrt{K_{\Psi}(z, z)}} \rightarrow 0
$$

as $|z| \rightarrow+\infty$. But this holds trivially because $K_{\Psi}(z, z)$ is an infinite power series in $|z|^{2}$ with positive coefficients.

Lemma 6.2. Let $f: \mathbb{C}^{n} \rightarrow \mathbb{C}$ be a function for which there exist positive numbers $R$ and $\varepsilon$ such that

$$
|f(z)-f(w)| \leq \varepsilon \varrho(z, w)
$$

whenever $|z| \geq R$. Then there exists a function $f_{0}: \mathbb{C}^{n} \rightarrow \mathbb{C}$ such that $f(z)=f_{0}(z)$ for $|z| \geq R$ and

$$
\left|f_{0}(z)-f_{0}(w)\right| \leq \varepsilon \varrho(z, w)
$$

for all points $z$ and $w$ in $\mathbb{C}^{n}$.

Proof. We argue as in the proof of Lemma 5.1 in [1]. We assume without loss of generality that $f$ is real-valued and set

$$
f_{0}(z):=\inf _{w \in \mathbb{C}^{n}}\{f(w)+\varepsilon \varrho(z, w)\} .
$$

Then a straightforward argument using the triangle inequality for the Bergman metric shows that $f_{0}$ has the desired properties.

Proof of Theorem $C$. We first prove the implication (i) $\Rightarrow$ (ii). Assuming that $H_{\bar{f}}$ is compact, we obtain, using Lemma 6.1 that

$$
[(\operatorname{MO} f)(z)]^{2}=\frac{\left\|H_{\bar{f}} K_{\Psi}(\cdot, z)\right\|^{2}}{K_{\Psi}(z, z)} \rightarrow 0
$$

when $|z| \rightarrow+\infty$. This gives the desired conclusion.

We next note that the implication (ii) $\Rightarrow$ (iii) is immediate from (2.2).

Finally, to prove that (iii) implies (i), in view of Theorem A, we only need to prove that the bounded Hankel operator $H_{\bar{f}}$ is compact whenever (iii) is satisfied. To see that this holds, we choose an arbitrary positive $\varepsilon$. Assuming (iii), we may find a positive $R_{0}$ such that

$$
|\langle(\nabla f)(z), \bar{\xi}\rangle| \leq \frac{\varepsilon}{2} \beta(z, \xi)
$$


whenever $|z| \geq R_{0}$ and $\xi$ is in $\mathbb{C}^{n} \backslash\{0\}$. Then for some $R>R_{0}$ we have

$$
|f(z)-f(w)| \leq \varepsilon \varrho(z, w)
$$

as long as $|z| \geq R$. Indeed, this follows because $\beta(z, \xi) /|\xi| \rightarrow \infty$ when $|z| \rightarrow \infty$ so that, whenever $|z|$ is sufficiently large, $\varrho(z, w)$ is "essentially" determined by the contribution to the integral in (1.2) from the points that lie outside the ball of radius $R_{0}$ centered at 0 . Now let $f_{0}$ be the function obtained from Lemma 6.2. We write

$$
H_{\bar{f}}=H_{\bar{f}-\bar{f}_{0}}+H_{\bar{f}_{0}}
$$

and observe that $\bar{f}-\bar{f}_{0}$ is a compactly supported continuous function on $\mathbb{C}^{n}$. Hence $H_{\bar{f}-\bar{f}_{0}}$ is compact. On the other hand, if $g$ a holomorphic polynomial, then

$$
\begin{aligned}
\left|H_{\bar{f}_{0}} g(z)\right| & \lesssim \int_{\mathbb{C}^{n}}\left|\bar{f}_{0}(w)-\bar{f}_{0}(z)\right|\left|K_{\Psi}(z, w) g(w)\right| d \mu_{\Psi}(w) \\
& \leq \varepsilon \int_{\mathbb{C}^{n}} \beta(z, \xi)\left|K_{\Psi}(z, w) g(w)\right| d \mu_{\Psi}(w)
\end{aligned}
$$

so that, by the proof of Theorem A, we see that $\left\|H_{\bar{f}_{0}}\right\| \lesssim \varepsilon$. The implication (iii) $\Rightarrow$ (i) follows because $\varepsilon$ can be chosen arbitrarily small.

\section{THE GEOMETRY OF BERGMAN BALLS OF FIXED RADIUS}

In what follows, we will need the analogue of Lemma 3.2 for the function $\Psi$ when $n>1$. We will therefore assume that

$$
\Psi^{\prime \prime}(t)=O\left(t^{-\frac{1}{2}}\left[\Psi^{\prime}(t)\right]^{1+\eta}\right) \quad \text { when } t \rightarrow \infty
$$

for some $\eta<1 / 2$ whenever $n>1$. This is again a mild smoothness condition on $\Psi$.

Lemma 7.1. Assume that (7.1) holds for some $\eta<1 / 2$. Then, for any fixed $\alpha>\eta$, we have

$$
\sup _{|\tau| \leq t^{1 / 2}\left[\Psi^{\prime}(t)\right]^{-\alpha}} \Psi^{\prime}(t+\tau)=(1+o(1)) \Psi^{\prime}(t)
$$

when $t \rightarrow \infty$.

We are interested in describing geometrically the Bergman ball

$$
B(z, a)=\{w: \varrho(z, w)<a\} .
$$

Let $P_{z}$ denote the orthogonal projection in $\mathbb{C}^{n}$ onto the complex line $\{\zeta z: \zeta \in \mathbb{C}\}$, where $z$ is an arbitrary point in $\mathbb{C}^{n} \backslash\{0\}$. It will be convenient to let $P_{0}$ denote the identity map. We use the notation

$$
D(z, a)=\left\{w:\left|z-P_{z} w\right| \leq a\left[\Phi^{\prime}\left(|z|^{2}\right)\right]^{-1 / 2},\left|w-P_{z} w\right| \leq a\left[\Psi^{\prime}\left(|z|^{2}\right)\right]^{-1 / 2}\right\} .
$$

Then we have the following result. 
Lemma 7.2. Suppose that there exists a real number $\eta<1 / 2$ such that (1.4) holds and that (7.1) holds if $n>1$. Then, for every positive number a, there exist two positive numbers $m$ and $M$ such that

$$
D(z, m) \subset B(z, a) \subset D(z, M)
$$

for every $z$ in $\mathbb{C}^{n}$.

Proof. It suffices to prove that

$$
\varrho(z, w) \simeq\left|z-P_{z} w\right|\left[\Phi^{\prime}\left(|z|^{2}\right)\right]^{1 / 2}+\left|w-P_{z} w\right|\left[\Psi^{\prime}\left(|z|^{2}\right)\right]^{1 / 2}
$$

for $w$ in $D(z, M)$ for any fixed positive number $M$. (The latter term vanishes and can be disregarded when $n=1$.) To begin with, we note that Theorem $\mathrm{B}$ gives that

$$
\varrho(z, w) \simeq \inf _{\gamma} \int_{0}^{1}\left(\left|\gamma^{\prime}(t)\right|\left[\Psi^{\prime}\left(|\gamma(t)|^{2}\right)\right]^{1 / 2}+\left|\left\langle\gamma(t), \gamma^{\prime}(t)\right\rangle\right|\left[\Psi^{\prime \prime}\left(|\gamma(t)|^{2}\right)\right]^{1 / 2}\right) d t
$$

where the infimum is taken over all piecewise smooth curves $\gamma:[0,1] \rightarrow \mathbb{C}^{n}$ such that $\gamma(0)=z$ and $\gamma(1)=w$. If we choose $\gamma$ to be the line segment from $z$ to $P_{z} w$ followed by the line segment from $P_{z} w$ to $w$ and use that $\Psi^{\prime \prime}(x)=o\left(\left[\Psi^{\prime}(x)\right]^{1 / 2}\right)$ on the latter part of $\gamma$, we get from (7.3) that

$$
\varrho(z, w) \lesssim\left|z-P_{z} w\right|\left[\Phi^{\prime}\left(|z|^{2}\right)\right]^{1 / 2}+\left|P_{z} w-w\right|\left[\Psi^{\prime}\left(|z|^{2}\right)\right]^{1 / 2}+\left|P_{z} w-w\right|^{2} o\left(\Psi^{\prime}\left(|z|^{2}\right)\right) .
$$

This gives the desired bound from above because, by assumption, $\left|P_{z} w-w\right| \leq M\left[\Psi^{\prime}\left(|z|^{2}\right)\right]^{-1 / 2}$.

To prove the bound from below, we argue in the following way. Let $\ell(\gamma)$ denote the Euclidean length of $\gamma$. Set

$$
\varrho_{\gamma}^{*}(z, w)=\int_{0}^{1}\left(\left|\gamma^{\prime}(t)\right|\left[\Psi^{\prime}\left(|\gamma(t)|^{2}\right)\right]^{1 / 2}+\left|\left\langle\gamma(t), \gamma^{\prime}(t)\right\rangle\right|\left[\Psi^{\prime \prime}\left(|\gamma(t)|^{2}\right)\right]^{1 / 2}\right) d t
$$

and $\varrho^{*}(z, w)=\inf _{\gamma} \varrho_{\gamma}^{*}(z, w)$. We observe that (7.3) implies that

$$
\varrho(z, w) \gtrsim \inf _{t}\left[\Psi^{\prime}\left(|\gamma(t)|^{2}\right)\right]^{1 / 2} \ell(\gamma)
$$

whenever, say, $\varrho_{\gamma}^{*}(z, w) \leq 2 \varrho^{*}(z, w)$. Since we know by the first part of the proof that $\varrho(z, w) \lesssim$ 1 , this implies that

$$
\ell(\gamma) \lesssim \inf _{t}\left[\Psi^{\prime}\left(|\gamma(t)|^{2}\right)\right]^{-1 / 2}
$$

By Lemma 7.1, we therefore have

$$
\ell(\gamma) \lesssim\left[\Psi^{\prime}\left(|z|^{2}\right)\right]^{-1 / 2}
$$

which, in view of (7.4), in turn gives

$$
\ell(\gamma) \lesssim\left[\Psi^{\prime}\left(|z|^{2}\right)\right]^{-1 / 2} \varrho(z, w)
$$

Now let $\gamma$ be any curve such that $\varrho_{\gamma}^{*}(z, w) \leq 2 \varrho^{*}(z, w)$. We then get from (7.3) that

$$
\varrho(z, w) \gtrsim|z-w|\left[\Psi^{\prime}\left(|z|^{2}\right)\right]^{1 / 2}+\int_{0}^{1}\left|\left\langle\gamma(t), \gamma^{\prime}(t)\right\rangle\right|\left[\Psi^{\prime \prime}\left(|\gamma(t)|^{2}\right)\right]^{1 / 2} d t .
$$


Set $\gamma_{0}(t)=P_{z}(\gamma(t))$ and $\gamma_{1}(t)=\gamma(t)-\gamma_{0}(t)$. Note that $\gamma_{1}(0)=0$ and that $\ell\left(\gamma_{1}\right) \leq \ell(\gamma)$. By orthogonality and the triangle inequality, we get

$$
\begin{aligned}
\int_{0}^{1}\left|\left\langle\gamma(t), \gamma^{\prime}(t)\right\rangle\right|\left[\Psi^{\prime \prime}\left(|\gamma(t)|^{2}\right)\right]^{1 / 2} d t \geq & \int_{0}^{1}\left|\gamma_{0}(t)\right|\left|\gamma_{0}^{\prime}(t)\right|\left[\Psi^{\prime \prime}\left(\left|\gamma_{0}(t)\right|^{2}\right)\right]^{1 / 2} d t \\
& -\int_{0}^{1}\left|\left\langle\gamma_{1}(t), \gamma_{1}^{\prime}(t)\right\rangle\right|\left[\Psi^{\prime \prime}\left(|\gamma(t)|^{2}\right)\right]^{1 / 2} d t
\end{aligned}
$$

Let $t_{1}$ be the smallest $t$ such that $\left|z-\gamma_{0}(t)\right|=\left|z-P_{z} w\right|$. Using that $\Psi^{\prime \prime}(x)=o\left(\left[\Psi^{\prime}(x)\right]^{2}\right)$ and (7.5), we then get

$$
\begin{aligned}
\int_{0}^{1}\left|\left\langle\gamma(t), \gamma^{\prime}(t)\right\rangle\right|\left[\Psi^{\prime \prime}\left(|\gamma(t)|^{2}\right)\right]^{1 / 2} d t & \geq(1+o(1)) \int_{0}^{t_{1}}|z|\left|\gamma_{0}^{\prime}(t)\right|\left[\Psi^{\prime \prime}\left(|z|^{2}\right)\right]^{1 / 2} d t-[\ell(\gamma)]^{2} o\left(\Psi^{\prime}\left(|z|^{2}\right)\right) \\
& \gtrsim\left|z-P_{z} w \| z\right|\left[\Psi^{\prime \prime}\left(|z|^{2}\right)\right]^{1 / 2}-o(1) \varrho(z, w)
\end{aligned}
$$

when $|z| \rightarrow \infty$. Plugging this estimate into (7.6), we obtain the desired bound from below.

It follows from the previous lemma that the Euclidean volume of $B(z, r)$ can be estimated as

$$
|B(z, r)| \simeq\left[\Phi^{\prime}\left(|z|^{2}\right)\right]^{-1 / 2}\left[\Psi^{\prime}\left(|z|^{2}\right)\right]^{(n-1) / 2}
$$

when $r$ is a fixed positive number. We will now use this fact to establish two covering lemmas.

Lemma 7.3. Suppose that there exists a real number $\eta<1 / 2$ such that (1.4) holds and that (7.1) holds if $n>1$. Let $R$ be a positive number and $m$ a positive integer. Then there exists a positive integer $N$ such that every Bergman ball $B(a, r)$ with $r \leq R$ can be covered by $N$ Bergman balls $B\left(a_{k}, \frac{r}{m}\right)$.

Proof. Fix a ball $B(a, r)$. Choose $a_{0}:=a$ and let $a_{1}$ be a point in $\mathbb{C}^{n}$ such that $\varrho\left(a, a_{1}\right)=r / m$. Now iterate so that in the $k$-th step $a_{k}$ is chosen as a point in the complement of $\bigcup_{j=1}^{k-1} B\left(a_{j}, r / m\right)$ minimizing the distance from $a$, and let $J$ be the smallest $k$ such that $\varrho\left(a, a_{k}\right) \geq r$. Then the balls $B\left(a_{0}, r / m\right), \ldots, B\left(a_{J-1}, r / m\right)$ constitute a covering of $B(a, r)$. By the triangle inequality, we see that the sets $B\left(a_{j}, r /(2 m)\right)$ are mutually disjoint, and they are all contained in $B(a, r+r /(2 m))$ when $j<J$. Hence

$$
\sum_{j=0}^{J-1}\left|B\left(a_{j}, r /(2 m)\right)\right| \leq|B(a, r+r /(2 m))| .
$$

On the other hand, by (7.7), it follows that there is a positive number $C$ depending on $R$ and $m$ but not on $a$ such that

$$
\frac{1}{C}|B(a, r+r /(2 m))| \leq\left|B\left(a_{j}, r /(2 m)\right)\right|
$$

for every $j$. We observe that it suffices to take $N$ to be the smallest positive integer larger than or equal to $C$.

Inspired by the construction in the previous lemma, we introduce the following notion. We say that a sequence of distinct points $\left(a_{k}\right)$ in $\mathbb{C}^{n}$ is a $\Psi$-lattice if the there exists a positive number $r$ such that the balls $B\left(a_{k}, r\right)$ constitute a covering of $\mathbb{C}^{n}$ and the balls $B\left(a_{k}, r / 2\right)$ are mutually disjoint. Replacing $a$ by, say, 0 and $r / m$ by $r$ in the previous proof, we have a straightforward 
way of constructing a $\Psi$-lattice. Note that since the balls $B\left(a_{k}, r / 2\right)$ are mutually disjoint, we must have $\varrho\left(a_{k}, a_{j}\right) \geq r$ when $k \neq j$. The number $r$, which may fail to be unique, is called a covering radius for the $\Psi$-lattice $\left(a_{k}\right)$. The supremum of all the covering radii is again a covering radius; it will be called the maximal covering radius for $\left(a_{k}\right)$.

Lemma 7.4. Suppose that there exists a real number $\eta<1 / 2$ such that (1.4) holds and that (7.1) holds if $n>1$, and let $R$ be a positive number. Then there exists a positive integer $N$ such that if $\left(a_{k}\right)$ is a $(\Psi)$-lattice with maximal covering radius $r \leq R / 2$, then every point $z$ in $\mathbb{C}^{n}$ belongs to at most $N$ of the sets $B\left(a_{k}, 2 r\right)$.

Proof. Let $N$ be the integer obtained from Lemma 7.3 for the given $R$ when $m=4$ and assume that $z \in \bigcap_{j=1}^{N+1} B\left(a_{k_{j}}, 2 r\right)$. Then $a_{k_{j}}$ is in $B(z, 2 r)$ for every $j=1, \cdots, N+1$. If the sets $B\left(z_{1}, r / 2\right), \ldots, B\left(z_{N}, r / 2\right)$ constitute a covering of $B(z, 2 r)$, the existence of which is guaranteed by Lemma 7.3, then at least one of the sets $B\left(z_{k}, r / 2\right)$ must contain two of the points $a_{k_{j}}, j=$ $1, \cdots, N+1$. On the other hand, by the triangle inequality, we have reached a contradiction because the minimal distance between any two points in the sequence $\left(a_{k}\right)$ can not be smaller than $r$.

\section{CARLESON MEASURES AND TOEPLitZ OPERATORS}

For a nonnegative Borel measure $\nu$ on $\mathbb{C}^{n}$, we set

$$
d \nu_{\Psi}(z)=e^{-\Psi\left(|z|^{2}\right)} d \nu(z) .
$$

Such a measure $\nu$ is called a Carleson measure for $\mathcal{A}^{2}(\Psi)$ if there is a positive constant $C$ such that

$$
\int_{\mathbb{C}^{n}}|f(z)|^{2} d \nu_{\Psi}(z) \leq C \int_{\mathbb{C}^{n}}|f(z)|^{2} d \mu_{\Psi}(z)
$$

for every function $f$ in $\mathcal{A}^{2}(\Psi)$. Thus $\nu$ is a Carleson measure for $\mathcal{A}^{2}(\Psi)$ if and only if the embedding $E_{\nu}$ of $\mathcal{A}^{2}(\Psi)$ into the space $L^{2}\left(\nu_{\Psi}\right)$ is bounded.

Theorem D. Let $\Psi$ be a logarithmic growth function, and suppose that there exists a real number $\eta<1 / 2$ such that (1.4) holds and that (7.1) holds if $n>1$. If $\nu$ is a nonnegative Borel measure on $\mathbb{C}^{n}$, then the following statements are equivalent:

(i) $\nu$ is a Carleson measure for $\mathcal{A}^{2}(\Psi)$;

(ii) There is a constant $C>0$ such that

$$
\int_{\mathbb{C}^{n}} \frac{\left|K_{\Psi}(w, z)\right|^{2}}{K(z, z)} d \nu_{\Psi}(w) \leq C
$$

for every $z$ in $\mathbb{C}^{n}$;

(iii) For every positive number $r$, there is a positive number $C$ such that

$$
\nu(B(z, r)) \leq C|B(z, r)|
$$

for every $z$ in $\mathbb{C}^{n}$; 
(iv) There exist a $\Psi$-lattice $\left(a_{k}\right)$ and a positive number $C$ such that

$$
\nu\left(B\left(a_{k}, r\right)\right) \leq C\left|B\left(a_{k}, r\right)\right|
$$

for every point $k$, where $r$ is the maximal covering radius for $\left(a_{k}\right)$.

We prepare for the proof of Theorem D by establishing the following two lemmas.

Lemma 8.1. Suppose that there exists a real number $\eta<1 / 2$ such that (1.4) holds and that (7.1) holds if $n>1$. Then there exists a positive number $r_{0}$ such that

$$
\left|K_{\Psi}(z, w)\right|^{2} \simeq K(z, z) K(w, w)
$$

holds for $z$ and $w$ whenever $\varrho(z, w) \leq r_{0}$.

Proof. The lemma follows from Lemma 3.1 along with Lemma 7.2 .

Lemma 8.2. Suppose that there exists a real number $\eta<1 / 2$ such that (1.4) holds and that (7.1) holds if $n>1$, and let $r_{0}$ be the constant from Lemma 8.1] Then there is a constant $C$ such that

$$
|f(z)|^{2} e^{-\Psi\left(|z|^{2}\right)} \leq \frac{C}{|B(z, r)|} \int_{B(z, r)}|f(w)|^{2} d \mu_{\Psi}(w)
$$

for every entire function $f$ on $\mathbb{C}^{n}$ and every $z$ in $\mathbb{C}^{n}$.

Proof. By Lemma 8.1, the holomorphic function $w \mapsto K(z, w)$ does not vanish at any point in $B(z, r)$. Thus the function $w \mapsto|f(w)|^{2}\left|K_{\Psi}(z, w)\right|^{-2}$ is subharmonic in $B(z, r)$. Choosing $m$ as in Lemma 7.2, we therefore get

$$
\begin{aligned}
|f(z)|^{2}|K(z, z)|^{-2} & \lesssim \frac{1}{|D(z, m)|} \int_{D(z, m)}|f(w)|^{2}\left|K_{\Psi}(z, w)\right|^{-2} d V(w) \\
& \lesssim \frac{1}{|B(z, r)|} \int_{B(z, r)}|f(w)|^{2}\left|K_{\Psi}(z, w)\right|^{-2} d V(w) .
\end{aligned}
$$

Applying Lemma 8.1 to the integrand to the left and then Lemma 3.1 to each side, we arrive at the desired estimate.

Note that, by (7.7), the lemma is valid for all positive $r$, with the additional proviso that $C$ depend on $r$.

Proof of Theorem $D$. We begin by noting that the implication (i) $\Rightarrow$ (ii) is trivial because it is just the statement that the Carleson measure condition holds for the functions $K(\cdot, z)$. To prove that (ii) implies (iii), we assume that (ii) holds and consider a ball $B(z, r)$ where $r$ is a fixed positive number. Then, by Lemma 8.1 and (7.7), we have

$$
\frac{1}{|B(z, r)|} \lesssim \frac{\left|K_{\Psi}(z, w)\right|^{2}}{K_{\Psi}(z, z)} e^{-\Psi\left(|w|^{2}\right)}
$$

when $\varrho(z, w) \leq r_{0}$, and therefore we obtain

$$
\frac{\nu(B(z, r))}{|B(z, r)|} \lesssim \int_{\mathbb{C}^{n}} \frac{\left|K_{\Psi}(z, w)\right|^{2}}{K_{\Psi}(z, z)} e^{-\Psi\left(|w|^{2}\right)} d \nu(w) \leq C .
$$


The implication (iii) $\Rightarrow$ (iv) is trivial (modulo the existence of $\Psi$-lattices), and we are therefore done if we can prove that (iv) implies (i). To this end, assume that (iv) holds, and let $\left(a_{k}\right)$ be a $\Psi$-lattice with maximal covering radius $r$. By Lemma 8.2, we see that

$$
\sup _{z \in B\left(a_{k}, r\right)}|f(z)|^{2} e^{-\Psi\left(|z|^{2}\right)} \lesssim \frac{1}{\left|B\left(a_{k}, 2 r\right)\right|} \int_{B\left(a_{k}, 2 r\right)}|f(w)|^{2} d \mu_{\Psi}(z)
$$

for every $k$. We therefore get

$$
\int_{\mathbb{C}^{n}}|f(z)|^{2} d \nu_{\Psi}(z) \lesssim \sum_{k} \int_{B\left(a_{k}, 2 r\right)}|f(w)|^{2} d \mu_{\Psi}(w) \lesssim \int_{\mathbb{C}^{n}}|f(w)|^{2} d \mu_{\Psi}(w),
$$

where the latter inequality holds by Lemma 7.4 .

For $\nu$ a nonnegative Borel measure on $\mathbb{C}^{n}$, we define the Toeplitz operator $T_{\nu}$ on $\mathcal{A}^{2}(\Psi)$ in the following way:

$$
\left(T_{\nu} f\right)(z):=\int_{\mathbb{C}^{n}} f(w) K_{\Psi}(z, w) e^{-\Psi\left(|w|^{2}\right)} d \nu(w) .
$$

A computation shows that $E_{\nu}^{*} E_{\nu}=T_{\nu}$. Thus Theorem D characterizes bounded Toeplitz operators. Compact Toeplitz operators can likewise be characterized via so-called vanishing Carleson measures; an obvious and straightforward modification of Theorem D gives a description of such measures. Toeplitz operators belonging to the Schatten classes $\mathcal{S}_{p}$ are characterized by the following theorem.

Theorem E. Let $\Psi$ be a logarithmic growth function, and suppose that there exists a real number $\eta<1 / 2$ such that (1.4) holds and that (7.1) holds if $n>1$. If $\nu$ is a nonnegative Borel measure on $\mathbb{C}^{n}$ and $p \geq 1$, then the following statements are equivalent:

(i) The Toeplitz operator $T_{\nu}$ on $\mathcal{A}^{2}(\Psi)$ belongs to the the Schatten class $\mathcal{S}_{p}$;

(ii) There exists a $\Psi$-lattice $\left(a_{k}\right)$ such that

$$
\sum_{k=1}^{\infty}\left(\frac{\nu\left(B\left(a_{k}, r\right)\right)}{\left|B\left(a_{k}, r\right)\right|}\right)^{p}<+\infty
$$

where $r$ is the maximal covering radius for $\left(a_{k}\right)$.

For the proof of this theorem, we require the following two lemmas.

Lemma 8.3. Suppose that $\left(e_{j}\right)$ is an orthonormal basis for $\mathcal{A}^{2}(\Psi)$ and that $\left(a_{j}\right)$ is a $\Psi$-lattice. Then the operator $J$ on $\mathcal{A}^{2}(\Psi)$ defined by

$$
J e_{j}(z):=\frac{K_{\Psi}\left(z, a_{j}\right)}{\sqrt{K_{\Psi}\left(a_{j}, a_{j}\right)}}
$$

is bounded.

Proof. For two arbitrary functions $f=\sum_{j} c_{j} e_{j}$ and $g$ in $\mathcal{A}^{2}(\Psi)$, the reproducing formula and the Cauchy-Schwarz inequality give

$$
|\langle J f, g\rangle|^{2}=\left|\sum_{j} c_{j} \frac{\overline{g\left(a_{j}\right)}}{\sqrt{K_{\Psi}\left(a_{j}, a_{j}\right)}}\right|^{2} \leq\left(\sum_{j}\left|c_{j}\right|^{2}\right)\left(\sum_{k} \frac{\left|g\left(a_{k}\right)\right|^{2}}{K_{\Psi}\left(a_{k}, a_{k}\right)}\right) .
$$


If we set

$$
\nu:=\sum_{k} \frac{e^{\Psi\left(\left|a_{j}\right|^{2}\right)}}{K_{\Psi}\left(a_{j}, a_{j}\right)} \delta_{a_{j}}
$$

then we may write this estimate as

$$
|\langle J f, g\rangle|^{2} \leq\|f\|_{\mathcal{A}^{2}(\Psi)}^{2} \int_{\mathbb{C}^{n}}|g(z)|^{2} d \nu_{\Psi}(z) .
$$

By Theorem $\mathrm{D}$, we see that $\nu$ is a Carleson measure, which implies that $J$ is a bounded operator on $\mathcal{A}^{2}(\Psi)$.

Lemma 8.4. Suppose that $T$ is a positive operator on $\mathcal{A}^{2}(\Psi)$. Then the trace of $T$ can be computed as

$$
\operatorname{Tr}(T)=\int_{\mathbb{C}^{n}} \widetilde{T}(z) K_{\Psi}(z, z) d \mu_{\Psi}(z)
$$

Proof. We write $K_{\Psi}(z, w)=\sum_{k=0}^{\infty} e_{k}(z) \overline{e_{k}(w)}$, where $\left(e_{k}\right)$ is an orthonormal basis for $\mathcal{A}^{2}(\Psi)$. The lemma is then proved by means of the following computation:

$$
\operatorname{Tr}(T)=\sum_{k=0}^{\infty}\left\langle T f_{k}, f_{k}\right\rangle_{\mathcal{A}^{2}(\Psi)}=\int_{\mathbb{C}^{n}}\left\langle T K_{\Psi}(\cdot, z), K_{\Psi}(\cdot, z)\right\rangle_{\mathcal{A}^{2}(\Psi)} d \mu_{\Psi}(z) .
$$

Proof of Theorem E. We begin by assuming that $T_{\nu}$ is in $\mathcal{S}_{p}$. Pick a $\Psi$-lattice $\left(a_{j}\right)$ and let $r$ be its maximal covering radius. By (7.7) and Lemma 8.1, we have

$$
\begin{aligned}
\sum_{k}\left(\frac{\nu\left(B\left(a_{k}, r\right)\right)}{\left|B\left(a_{k}, r\right)\right|}\right)^{p} & \simeq \sum_{k}\left(\int_{B\left(a_{k}, r\right)} K_{\Psi}(w, w) d \nu_{\Psi}(w)\right)^{p} \\
& \simeq \sum_{k}\left(\int_{B\left(a_{k}, r\right)} \frac{\left|K_{\Psi}\left(a_{k}, w\right)\right|^{2}}{K_{\Psi}\left(a_{k}, a_{k}\right)} d \nu_{\Psi}(w)\right)^{p}
\end{aligned}
$$

By Lemma 7.4 and our assumption on $\nu$, this gives

$$
\sum_{k}\left(\frac{\nu\left(B\left(a_{k}, r\right)\right)}{\left|B\left(a_{k}, r\right)\right|}\right)^{p} \lesssim \sum_{k}\left(\int_{\mathbb{C}^{n}} \frac{\left|K_{\Psi}\left(a_{k}, w\right)\right|^{2}}{K_{\Psi}\left(a_{k}, a_{k}\right)} d \mu_{\Psi}(w)\right)^{p} .
$$

If we construct $J$ as in Lemma 8.3 , then the right-hand side equals $\sum_{k}\left|\left\langle J^{*} T_{\nu} J e_{k}, e_{k}\right\rangle\right|^{p}$. Since $J$ is a bounded operator, $J^{*} T_{\nu} J$ also belongs to $\mathcal{S}_{p}$, and so the latter sum converges. We conclude that (i) implies (ii).

We will use an interpolation argument to prove that (ii) implies (i). We already know from Theorem $\mathrm{D}$ that $T_{\nu}$ is in the Schatten class $\mathcal{S}_{\infty}$ whenever $\nu\left(B\left(a_{k}, r\right)\right) \leq C\left|B\left(a_{k}, r\right)\right|$ for some positive constant $C$. Suppose now that

$$
\sum_{k} \frac{\nu\left(B\left(a_{k}, r\right)\right)}{\left|B\left(a_{k}, r\right)\right|}<+\infty
$$


and let $\left(e_{j}\right)$ be an orthonormal basis for $\mathcal{A}^{2}(\Psi)$. By the reproducing formula, we have

$$
\left\langle T_{\nu} e_{j}, e_{j}\right\rangle=\int_{\mathbb{C}^{n}}\left|e_{j}(w)\right|^{2} d \nu_{\Psi}(w)
$$

which implies that

$$
\sum_{j}\left|\left\langle T_{\nu} e_{j}, e_{j}\right\rangle\right|=\int_{\mathbb{C}^{n}} K_{\Psi}(w, w) d \nu_{\Psi}(w) \leq \sum_{k} \int_{B\left(a_{k}, r\right)} K_{\Psi}(w, w) d \nu_{\Psi}(w) .
$$

Using again Lemma 3.1, we then get

$$
\sum_{j}\left|\left\langle T_{\nu} e_{j}, e_{j}\right\rangle\right| \lesssim \sum_{k} \frac{\nu\left(B\left(a_{k}, r\right)\right)}{\left|B\left(a_{k}, r\right)\right|}<+\infty,
$$

which means that $T_{\nu}$ belongs to $\mathcal{S}_{1}$. By interpolation, we conclude that (ii) implies (i).

We remark that the theorems proved in this section generalize results for the classical Fock space when $n=1$ obtained recently in [12]. It may be noted that Theorem $\mathrm{D}$ above could be elaborated to include two additional conditions for membership in $\mathcal{S}_{p}$, in accordance with Theorem 4.4 in [12]. The proof would be essentially the same as the proof of the latter theorem. Note that [12] also treats Schatten class membership of Toeplitz operators for $p<1$.

\section{SCHATTEN CLASS MEMBERShiP OF HANKEL OPERATORS}

Our work so far suggests two possible definitions of Besov spaces, in accordance with our respective definitions of $\operatorname{BMOA}(\Psi)$ and $\mathcal{B}(\Psi)$. We let $\mathcal{B}_{m}^{p}(\Psi)$ denote the set of entire functions $f$ such that

$$
\int_{\mathbb{C}^{n}}[\operatorname{MO} f(z)]^{p} K_{\Psi}(z, z) d \mu_{\Psi}(z)<\infty
$$

for a function $h: \mathbb{C}^{n} \rightarrow \mathbb{C}^{n}$, we set

$$
|h(z)|_{\beta}=\sup _{\xi \in \mathbb{C}^{n} \backslash\{0\}} \frac{|\langle h(z), \bar{\xi}\rangle|}{\beta(z, \xi)},
$$

and we let $\mathcal{B}_{d}^{p}(\Psi)$ be the set of entire functions $f$ for which

$$
\int_{\mathbb{C}^{n}}|\nabla f(z)|_{\beta}^{p} K_{\Psi}(z, z) d \mu_{\Psi}(z)<\infty .
$$

These definitions are in line with those of K. Zhu for Hankel operators on the Bergman space of the unit ball in $\mathbb{C}^{n}$ [19].

It is immediate from (2.2) that $\mathcal{B}_{m}^{p}(\Psi) \subset \mathcal{B}_{d}^{p}(\Psi)$. The basic question is whether these spaces coincide and in fact characterize Schatten class Hankel operators with anti-holomorphic symbols. The following theorem gives an affirmative answer to this question.

Theorem F. Let $\Psi$ be a logarithmic growth function, and suppose that there exists a real number $\eta<1 / 2$ such that (1.4) holds and that (7.1) holds if $n>1$. If $f$ is an entire function on $\mathbb{C}^{n}$ and $p \geq 2$, then the following statements are equivalent: 
(i) The function $f$ belongs to $\mathcal{T}(\Psi)$ and the Hankel operator $H_{\bar{f}}$ on $\mathcal{A}^{2}(\Psi)$ is in the Schatten class $\mathcal{S}_{p}$;

(ii) The function $f$ belongs to $\mathcal{B}_{m}^{p}(\Psi)$;

(iii) The function $f$ belongs to $\mathcal{B}_{d}^{p}(\Psi)$.

Proof. We have already observed that the implication (ii) $\Rightarrow$ (iii) is an immediate consequence of (2.2). The implication (i) $\Rightarrow$ (ii) relies on the following general Hilbert space argument. If (i) holds, then the operator $\left[H_{\bar{f}}^{*} H_{\bar{f}}\right]^{\frac{p}{2}}$ is in the trace class $\mathcal{S}_{1}$. Applying Lemma 8.4 and using the spectral theorem along with Hölder's inequality, we obtain

$$
\begin{aligned}
\operatorname{Tr}\left(\left[H_{\bar{f}}^{*} H_{\bar{f}}\right]^{\frac{p}{2}}\right) & =\int_{\mathbb{C}^{n}}\left\langle\left[H_{\bar{f}}^{*} H_{\bar{f}}\right]^{\frac{p}{2}} K_{\Psi}(\cdot, z), K_{\Psi}(\cdot, z)\right\rangle d \mu_{\Psi}(z) \\
& \gtrsim \int_{\mathbb{C}^{n}}\left[\frac{\left\|H_{\bar{f}} K_{\Psi}(\cdot, z)\right\|^{2}}{K_{\Psi}(z, z)}\right]^{\frac{p}{2}} K_{\Psi}(z, z) d \mu_{\Psi}(z) .
\end{aligned}
$$

Recalling the computation made in (2.1), we arrive at (ii).

Our proof of the implication (iii) $\Rightarrow$ (i) will use a version of L. Hörmander's $L^{2}$ estimates for the $\bar{\partial}$ operator. To this end, write $\Delta_{\Psi}(z)=\Psi\left(|z|^{2}\right)$ and observe that

$$
\alpha^{2}(z, \xi):=\sum_{j, k=1}^{n} \frac{\partial^{2} \Delta_{\Psi}(z)}{\partial z_{j} \partial \bar{z}_{k}} \xi_{j} \bar{\xi}_{k}=|\xi|^{2} \Psi^{\prime}\left(|z|^{2}\right)+|\langle z, \xi\rangle|^{2} \Psi^{\prime \prime}\left(|z|^{2}\right)
$$

for arbitrary vectors $z=\left(z_{1}, \ldots, z_{n}\right)$ and $\xi=\left(\xi_{1}, \ldots, \xi_{n}\right)$ in $\mathbb{C}^{n}$. By Theorem $\mathrm{B}$, we therefore have $\alpha(z, \xi) \simeq \beta(z, \xi)$. Now let $L_{\beta}^{2}\left(\mu_{\Psi}\right)$ be the space of vector valued functions $h=\left(h_{1}, \cdots h_{n}\right)$, identified with the corresponding $(0,1)$-forms $h_{1} d \bar{z}_{1}+\cdots+h_{n} d \bar{z}_{n}$ such that

$$
\|h\|_{L_{\beta}^{2}\left(\mu_{\Psi}\right)}^{2}:=\int_{\mathbb{C}^{n}}|h(z)|_{\beta}^{2} d \mu_{\Psi}(z)<\infty .
$$

It follows from Theorem 2.2 in [7] (a special case of a theorem proved by J.-P. Demailly in [10]) that the operator $S$ giving the canonical solution to the $\bar{\partial}$-problem is bounded from $L_{\beta}^{2}\left(\mu_{\Psi}\right)$ into $L^{2}\left(\mu_{\Psi}\right)$.

Since $f$ is holomorphic, we have

$$
\bar{\partial}\left(H_{\bar{f}} g\right)=\overline{\nabla f} g
$$

when $g$ is in $\mathcal{A}^{2}(\Psi)$, whence $H_{\bar{f}} g=S(\overline{\nabla f} g)$. Thus it follows that

$$
\left\|H_{\bar{f}} g\right\|_{L^{2}\left(\mu_{\Psi}\right)} \lesssim \int_{\mathbb{C}^{n}}|\nabla f(z)|_{\beta}^{2}|g(z)|^{2} d \mu_{\Psi}(z)
$$

If we set $d \nu(z)=|\nabla f(z)|_{\beta}^{2} d V(z)$, this may be written as

$$
H_{\bar{f}}^{*} H_{\bar{f}} \lesssim M_{|\nabla f|_{\beta}}^{*} M_{|\nabla f|_{\beta}}=T_{\nu}
$$


where as before $M_{h}$ denotes the operator of multiplication by $h$ from $\mathcal{A}^{2}(\Psi)$ into $L^{2}\left(\mu_{\Psi}\right)$. By Theorem E, it remains to verify that (iii) implies that for some $\Psi$-lattice $\left(a_{k}\right)$ we have

$$
\sum_{k=1}^{\infty}\left(\frac{\nu\left(B\left(a_{k}, r\right)\right)}{\left|B\left(a_{k}, r\right)\right|}\right)^{p / 2}<+\infty
$$

where $r$ is the maximal covering radius for $\left(a_{k}\right)$. To this end, we first observe that Hölder's inequality gives that

$$
\left(\frac{\nu(B(z, r))}{|B(z, r)|}\right)^{p / 2} \lesssim \frac{1}{|B(z, r)|} \int_{B(z, r)}|\nabla f(z)|_{\beta}^{p} d V(w) .
$$

Hence, using (7.7) and Lemma 3.1, we obtain

$$
\left(\frac{\nu(B(z, r))}{|B(z, r)|}\right)^{p / 2} \lesssim \int_{B(z, r)}|\nabla f(z)|_{\beta}^{p} K(z, z) d V(w) .
$$

Now choosing any $\Psi$-lattice $\left(a_{k}\right)$ and using Lemma 7.4, we arrive at (9.2).

Several remarks are in order. First note that 9.1 gives another proof of the implication (iii) $\Rightarrow$ (i) in Theorem A, subject to the additional smoothness condition (7.1). Second, as shown in [9], there are nontrivial Hankel operators in $\mathcal{S}_{p}$ only when $p>2 n$. This fact is easy to see from Theorem $\mathrm{F}$ when $n=1$, because then

$$
|\nabla f(z)|_{\beta} \simeq\left|f^{\prime}(z)\right|\left[\Phi^{\prime}\left(|z|^{2}\right)\right]^{-1 / 2},
$$

whence $f$ is in $\mathcal{B}_{d}^{p}(\Psi)$ if and only if

$$
\int_{\mathbb{C}}\left|f^{\prime}(z)\right|^{p}\left[\Phi^{\prime}\left(|z|^{2}\right)\right]^{1-p / 2} d V(z)<\infty .
$$

When $n>1$, the computation of $|\nabla f(z)|_{\beta}$ is less straightforward, but we always have

$$
|\nabla f(z)|\left[\Phi^{\prime}\left(|z|^{2}\right)\right]^{-1 / 2} \lesssim|\nabla f(z)|_{\beta} \lesssim|\nabla f(z)|\left[\Psi^{\prime}\left(|z|^{2}\right)\right]^{-1 / 2} .
$$

The estimate from above shows that the condition

$$
\int_{\mathbb{C}^{n}}|\nabla f(z)|^{p} \Phi^{\prime}\left(|z|^{2}\right)\left[\Psi^{\prime}\left(|z|^{2}\right)\right]^{n-1-p / 2} d V(z)<\infty
$$

is sufficient for $f$ to belong to $\mathcal{B}_{d}^{p}(\Psi)$, and the estimate from below shows that this is also necessary when $\Phi^{\prime} / \Psi^{\prime}$ is a bounded function. We conclude from (9.3) and (9.4) that if the growth of $\Psi^{\prime}$ is super-polynomial, then $\mathcal{B}_{d}^{p}(\Psi)$ is infinite-dimensional and contains all polynomials if and only if $p>2 n$. This is immediate when $n=1$, and it follows also when $n>1$ because

$$
\int_{0}^{\infty} \frac{\Psi^{\prime \prime}(t)}{\left[\Psi^{\prime}(t)\right]^{1+\delta}} d t \leq \frac{1}{\delta\left[\Psi^{\prime}(0)\right]^{\delta}}<\infty
$$

for every $\delta>0$. If, on the other hand, $\Psi$ is a polynomial, then $\Phi^{\prime} / \Psi^{\prime}$ is a bounded function, and one may use (9.4) and Theorem $\mathrm{F}$ to deduce Theorem $\mathrm{B}$ in [8].

It is not hard to check that if $f$ is a monomial and $n>1$, then

$$
|\nabla f(z)|_{\beta} \simeq|\nabla f(z)| \mid\left[\Psi^{\prime}\left(|z|^{2}\right)\right]^{-1 / 2}
$$


for $z$ belonging to a set of infinite volume measure. By Lemma 2.12 in [8] and Theorem F above, one may therefore conclude as in [8] that $\mathcal{B}_{d}^{p}(\Psi)$ is nontrivial only if $p>2 n$.

\section{REFERENCES}

[1] W. Bauer, Mean oscillation and Hankel operators on the Segal-Bargmann space, Integr. Equ. Oper. Theory 52 (2005), 1-15.

[2] W. Bauer, Hilbert-Schmidt Hankel operators on the Segal-Bargmann space, Proc. Amer. Math. Soc. 132 (2005), 2989-2996.

[3] F. Beatrous \& S.-Y. Li, Trace ideal criteria for operators of Hankel type, Illinois J. Math. 39 (1995), $723-754$.

[4] D. Békollé, C. A. Berger, L. Coburn \& K. Zhu, BMO in the Bergman metric on bounded symmetric domains, J. Funct. Anal. 93 (1990), 310-350.

[5] C. A. Berger \& L. Coburn, Toeplitz operators on the Segal-Bargmann space, Trans. Amer. Math. Soc. 301 (1987), 813-829.

[6] C. A. Berger, L. Coburn \& K. Zhu, Toeplitz Operators and Function Theory in n-Dimensions, Lecture Notes in Math. 1256, Springer, Berlin, 1987.

[7] B. Berndtsson \& P. Charpentier, A Sobolev mapping property of the Bergman kernel, Math. Z. 235 (2000), $1-10$.

[8] H. Bommier-Hato \& E. H. Youssfi, Hankel operators on weighted Fock spaces, Integr. Equ. Oper. Theory 59 (2007), 1-17.

[9] H. Bommier-Hato \& E. H. Youssfi, Hankel operators and the Stieltjes moment problem, J. Funct. Anal. 258 (2010), 978-998.

[10] J.-P. Demailly, Estimations $L^{2}$ pour l'opérateur $\bar{\partial}$ d'un fibré vectoriel holomorphe semi-positif au-dessus d'une variété kählérienne complète, Ann. Sci. École Norm. Sup. 15 (1982), 457-511.

[11] F. Holland \& R. Rochberg, Bergman kernel asymptotics for generalized Fock spaces, J. Anal. Math. 83 (2001), 207-242.

[12] J. Isralowitz \& K. Zhu, Toeplitz operators on the Fock space, Integr. Equ. Oper. Theory 66 (2010), $593-611$.

[13] S. Janson, J. Peetre \& R. Rochberg, Hankel forms and the Fock space, Rev. Mat. Iberoamericana 3 (1987), 61-138.

[14] W. Knirsch \& G. Schneider, Continuity and Schatten-von Neumann p-class membership of Hankel operators with antiholomorphic symbols on (generalized) Fock spaces, J. Math. Anal. Appl. 320 (2006), 403-414.

[15] T. L. Kriete III, Kernel functions and composition operators in weighted Bergman spaces, Studies on composition operators (Laramie, WY, 1996), 73-91, Contemp. Math. 213, Amer. Math. Soc., Providence, RI, 1998.

[16] J. Marzo \& J. Ortega-Cerdà, Pointwise estimates for the Bergman kernel of the weighted Fock space, J. Geom. Anal. 19 (2009), 890-910.

[17] K. Stroethoff, Hankel operators in the Fock space, Michigan Math. J. 39 (1992), 3-16.

[18] J. Xia \& D. Zheng, Standard deviation and Schatten class Hankel operators on the Segal-Bargmann space, Indiana Univ. Math. J. 53 (2004), 1381-1399.

[19] K. Zhu, Schatten class Hankel operators on the Bergman space of the unit ball, Amer. J. Math. 113 (1991), 147-167.

[20] K. Zhu, Operator Theory in Function Spaces, Marcel Dekker, New York, 1990.

Seip: Department of Mathematical Sciences, Norwegian University of Science and TechNOLOGY (NTNU), NO-7491 TRONDHEIM, NORWAY

E-mail address: seip@math.ntnu.no

Youssfi: LATP, U.M.R. C.N.R.S. 6632, CMI, Université de Provence, 39 Rue F-Joliot-Curie, 13453 Marseille CEDEX 13, FranCE

E-mail address: youssfi@gyptis.univ-mrs.fr 